\title{
BodyStylus: Freehand On-Body Design and Fabrication of Epidermal Interfaces
}

\author{
Narjes Pourjafarian \\ pourjafarian@cs.uni-saarland.de \\ Saarland University, Saarland \\ Informatics Campus, Saarbrücken, \\ Germany.
}

\section{Sahar Mavali}

mavali@cs.uni-saarland.de

Saarland University, Saarland

Informatics Campus, Saarbrücken, Germany.

\author{
Marion Koelle \\ koelle@cs.uni-saarland.de \\ Saarland University, Saarland \\ Informatics Campus, Saarbrücken, \\ Germany. \\ Konstantin Klamka \\ konstantin.klamka@tu-dresden.de \\ Interactive Media Lab Dresden, \\ Technische Universität Dresden, \\ Dresden, Germany.
}

\author{
Bruno Fruchard \\ fruchard@cs.uni-saarland.de \\ Saarland University, Saarland \\ Informatics Campus, Saarbrücken, \\ Germany. \\ Daniel Groeger \\ groeger@cs.uni-saarland.de \\ Saarland University, Saarland \\ Informatics Campus, Saarbrücken, \\ Germany.
}

Paul Strohmeier

strohmeier@cs.uni-saarland.de

Saarland University, Saarland

Informatics Campus, Saarbrücken,

Germany.
Jürgen Steimle

steimle@cs.uni-saarland.de

Saarland University, Saarland

Informatics Campus, Saarbrücken,

Germany.
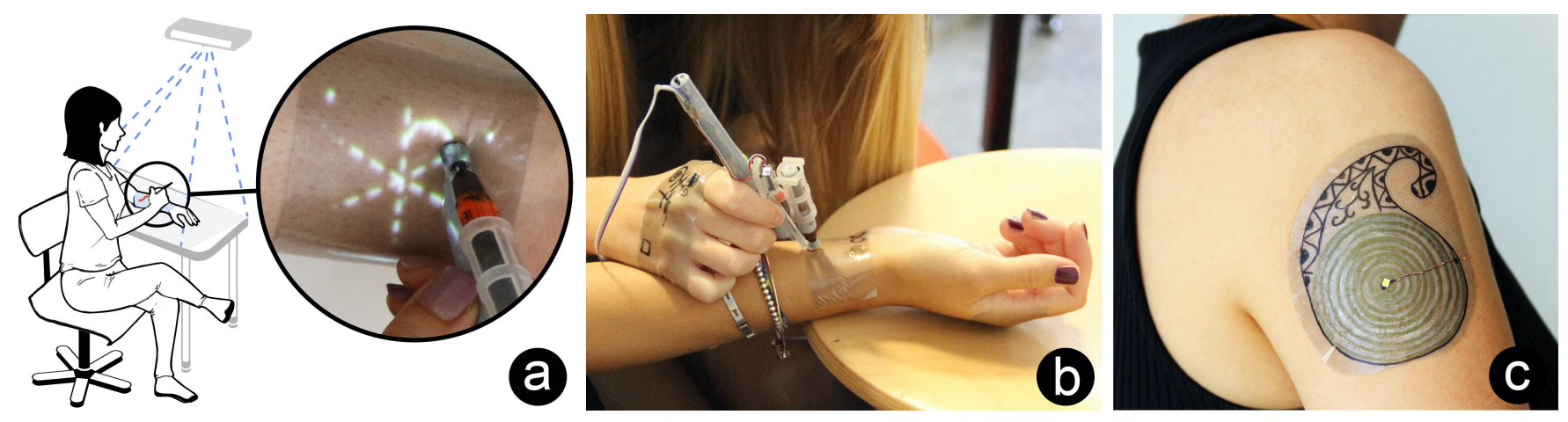

Figure 1: BodyStylus combines design and fabrication of functional on-body interfaces into one integrated activity performed directly on the body. A handheld device combines the ease and directness of free-form drawing with digital assistance; visual cues provide guidance while constraints dynamically restrict inking to prevent errors. BodyStylus enables drawing on one's own body (a), as well as collaborative design (b), to realize functional epidermal interfaces (c).

\footnotetext{
ABSTRACT

In traditional body-art, designs are adjusted to the body as they are applied, enabling creative improvisation and exploration. Conventional design and fabrication methods of epidermal interfaces, however, separate these steps. With BodyStylus we present the first computer-assisted approach for on-body design and fabrication of epidermal interfaces. Inspired by traditional techniques, we propose

Permission to make digital or hard copies of part or all of this work for personal or classroom use is granted without fee provided that copies are not made or distributed for profit or commercial advantage and that copies bear this notice and the full citation on the first page. Copyrights for third-party components of this work must be honored.

For all other uses, contact the owner/author(s).

CHI '21, May 8-13, 2021, Yokohama, Japan

(C) 2021 Copyright held by the owner/author(s)

ACM ISBN 978-1-4503-8096-6/21/05.

https://doi.org/10.1145/3411764.3445475
}

a hand-held tool that augments freehand inking with digital support: projected in-situ guidance assists creating valid on-body circuits and aesthetic ornaments that align with the human bodyscape, while pro-active switching between inking and non-inking creates error preventing constraints. We contribute BodyStylus's design rationale and interaction concept along with an interactive prototype that uses self-sintering conductive ink. Results of two focus group explorations showed that guidance was more appreciated by artists, while constraints appeared more useful to engineers, and that working on the body inspired critical reflection on the relationship between bodyscape, interaction, and designs.

\section{CCS CONCEPTS}

- Human-centered computing $\rightarrow$ Interaction devices. 


\section{KEYWORDS}

On-body design, on-body fabrication, epidermal devices, skin, wearable computing, craft, pen-based interaction

\section{ACM Reference Format:}

Narjes Pourjafarian, Marion Koelle, Bruno Fruchard, Sahar Mavali, Konstantin Klamka, Daniel Groeger, Paul Strohmeier, and Jürgen Steimle. 2021. BodyStylus: Freehand On-Body Design and Fabrication of Epidermal Interfaces. In CHI Conference on Human Factors in Computing Systems (CHI '21), May 8-13, 2021, Yokohama, Japan. ACM, New York, NY, USA, 15 pages. https://doi.org/10.1145/3411764.3445475

\section{INTRODUCTION}

The skin offers appealing properties as an input surface: it is large and always available, soft and deformable for expressive interaction beyond touch [98], while its tactile perception eases eyes-free interaction [20]. Prior research in and beyond HCI has explored a multiplicity of materials, fabrication techniques, and design approaches for exploring these properties in epidermal interfaces. They typically follow a three-step workflow: (1) designing the interface on a computer, e.g., using graphic design software [32, 46] or custom software tools [48, 56]; (2) fabricating the design, e.g., using screen printing [46], stencil or laser cutters [32, 48, 97], inkjet printers [35] or lamination techniques [48]; and eventually (3) applying the epidermal interface to the body, e.g., through water-transfer [46]. Recent work by Choi et al. [9] succeeds in merging steps 2 and 3 into one unified on-body fabrication step using an attachable printing device and conductive ink. Nevertheless, all of these approaches require an initial digital design step, separated from the body. In contrast, we wish to support the hands-on adaption of designs to the human body as is common in established body-based practices including tattooing, make-up, and henna.

Such direct on-body design enables fitting epidermal devices to the human bodyscape, e.g., making fine adjustments according to detailed shape and movement of the body, and supports in-situ creative exploration and expression. Pioneering work on computational on-body design and fabrication has so far been restricted to the fabrication of passive designs without interactive functionality [16, 17]. The addition of functional elements, however, is particularly challenging due to the complex interplay between circuitry, bodyscape, and aesthetics. Techniques from traditional on-body arts and crafts underpin the importance of a tight link between the design and the individual body size and shape. With this in mind, we aim to explore combined design and fabrication of functional epidermal devices directly on the body.

We present BodyStylus, the first computer-assisted approach for freehand on-body design and fabrication of epidermal devices. BodyStylus consists of a general concept, inspired by traditional body-art, a system including a handheld marker (Figure 1a), and a set of design techniques for creating epidermal interfaces. The handheld marker enables on-the-fly design, customization, and fabrication of epidermal interfaces directly on the user's body. The system supports the user in implementing functional devices respecting electronic constraints, aesthetics and body features: projected in-situ guidance facilitates functional and aesthetic results, while automatic switching between inking and non-inking modes prevents errors in circuit logic. We demonstrate the technical feasibility of BodyStylus with a proof-of-concept implementation using a custom-designed dispensing pen and self-sintering conductive ink to instantly create functional traces on epidermal devices.

The design techniques offer the user a rapid, direct, and handson way of creating visually aesthetic epidermal devices on the body. Based on varying levels of guidance and constraints, we offer techniques for inking functional conductive traces, creating aesthetic shapes and patterns, adding electronic components, and creating custom free-form components.

We show how these techniques can be used in concert to design and fabricate functional epidermal devices, alone or collaboratively, for various body locations. We demonstrate that BodyStylus reflects aesthetic and artisanal paradigms of traditional on-body arts and crafts, while also offering a means of facilitation and guidance orthogonal to traditional crafts.

In two focus groups, BodyStylus was practically explored with engineers and make-up artists. The findings uncover commonalities and differing perspectives as well as suggestions on how the practitioners would wish to incorporate it into their own workflow. The aspiring make-up artists were able to implement epidermal controls for special-effect LEDs within less than two hours after having learned about the concept of epidermal devices. Our observations further show that working on the body inspired critical reflection on the relationship between bodyscape, interaction, and design.

\section{RELATED WORK}

Our contribution builds on prior work on epidermal devices, interactive fabrication, sketch-based interfaces, and flexible electronics.

\subsection{Epidermal Devices}

Building on pioneering work on epidermal electronics [37], the $\mathrm{HCI}$ community has proposed using on-skin devices for interactive purposes [97]. Various forms of input and output on the epidermis have been investigated, including multi-touch input [56], visual feedback [32, 46, 95, 99], haptic output [22, 23, 52, 102, 105], stiffness change [31], and customized physiological sensing [55]. Fully wireless solutions can be realized [48].

The body has a complex geometry with many recognizable landmarks [88]. Such complex features of the body can be used to provide additional haptic guidance [99] and serve as mnemonic aids in interaction [3]. The importance of aesthetics for the social acceptability of body-worn interfaces has been emphasized early on. Cosmetics and traditional body decoration served as important sources of inspiration. For instance, Vega et al. [92] and Kao et al. [33] demonstrate how something as personal and customizable as makeup can be turned into an interface. Other work has presented approaches for the aesthetic design of on-skin electronics $[46,97]$ and functional tattoos [93]. In these works, even though various levels of customization exist, the design process is separated from the body and happens prior to the fabrication.

More recent work has started to investigate the social acceptability of on-skin devices [106, 107], a theme tightly coupled with body art and tattooing [75]. Along these lines, input (or inking) on someone else's body $[12,89]$ as well as the impact of interactive body 
markings on the wearer's social image $[40,106,107]$ are emerging areas of study. BodyStylus does not explicitly seek to investigate social issues around interactive body markings, but could serve as a tool to further investigate on-body co-creation and facilitate the design of socially acceptable on-body interfaces, by making the chosen body location (c.f., Harrison et al. [24]) more directly apparent during the creation process.

\subsection{Interactive and Handheld Fabrication}

Interactive fabrication allows for real-time manipulation of a fabrication device [51,101]. Our approach is inspired by concurrent interactive fabrication, where design and fabrication occur at the same time, often controlled with a handheld device. For instance, the influential FreeD device uses a handheld milling tool to sculpt and carve 3D models [110]. Further research leverages on handheld tools for various fabrication activities, such as cutting 2D surfaces [77], making 3D models [63, 64, 78, 90, 108], spray painting [85], or creating e-textiles [39].

A comparably small number of approaches focused on interactive fabrication around the body. Tactum [16] uses on-skin gestures to design 3D models directly on the body. ExoSkin [17], an important source of inspiration of our work, extends this approach to direct on-body fabrication using a custom handheld extruder. Both approaches are limited to fabricating passive geometries. In contrast, Zhu et al. [109] and Choi et al. [9] presented devices for printing conductors directly on the skin. However, they use a conventional CAD design-then-fabricate approach. We contribute to this line of work by presenting the first computer-assisted approach for direct on-body design and fabrication of epidermal electronics.

\subsection{Sketch-based Interfaces}

Sketching is a highly expressive medium [29]. Combining it with interactive user-interface tools gives the designer more flexibility and freedom to create design prototypes [43, 44]. A long history of research on sketching interfaces informed our use of visual realtime guides [103], pen-based position tracking [36, 38, 45, 104], and projected output [87]. Inspired by prior works and traditional bodyart practices, we use a sketch-based interface to assist the designer in drawing the epidermal device on-the-fly on the body.

\subsection{Prototyping of Flexible Electronics}

Conventional approaches for creating flexible electronics commonly involve techniques of printing [6, 34, 35, 58], patterning $[19,81]$, or textile processing $[21,66]$. To facilitate more rapid hands-on prototyping, researchers proposed using a conductive stylus or conductive paint [5, 49,53,67] or copper tape [81] directly on real-world objects. Prior research prominently highlighted the relevance of aesthetics in designing circuits $[5,71,72]$. Digitally designed tools for flexible electronics [26, 57, 58, 73] mainly focused on supporting novices with parametric electronic components to create electronic devices. We took inspiration from the direct handson fabrication of circuits using a stylus and digitally support users to create parametric components, but in contrast to this prior work, we directly design them on the body.

\section{DESIGN CONTEXT}

In this section, we take a close look at off-body and on-body work practices of established arts and crafts, and describe the design opportunities for BodyStylus that result from the unique combination of aesthetics, human bodyscape, and circuit logic.

\subsection{Draw on Aesthetically Rich Traditions}

Body markings are a time-honored and recurrent motif in the history of civilization: ocre body-paint on ritual performers, which might be considered one of the first expressions of human art, was used as early as 147,000 years ago to augment faces and bodies [96]. Permanent tattooing [15] and henna markings [60] have a long and complex history. Contemporary body markings include permanent tattoos of various styles [69], various forms of the non-permanent face and body paint, e.g., cosmetics, stage or carnival make-up, as well as semi-permanent drawn-on skin embellishments [27] from plant-based dyes, e.g., henna or jagua.

What many of these traditions share is that they work with handheld tools directly on the body and that the tools they use shape the aesthetics of the body marking and vice versa. For instance, depending on the desired aesthetic, a tattoo might be created using a tattoo machine vs. using so-called hand poking. In henna art, aesthetics evolved around what the available tools and materials afforded. Varying pressure applied to the henna applicator affects the geometry of the resulting shape, e.g., by creating a droplet. This tight coupling of the hands-on tool, ink, and skin contrasts with existing practices around on-skin devices, such as electronic tattoos, which are mostly designed and fabricated off-skin and only then applied.

BodyStylus builds upon these rich traditions of elaborating a design on the body by enabling the user to generate circuits using a pen on skin. Thereby, the process of fabricating on-body interfaces becomes more immediate and less detached from the body than CAD-based approaches. In its aesthetic, BodyStylus strongly draws on the intricate line art of henna, which, with its crisp lines and entwined ornaments is well suited for implementing electrical circuits.

\subsection{Relate Bodyscape, Ornament and Function}

Many traditional on-body arts and crafts incorporate the individuality of the human body into their practices: designs are adjusted to the unique size and shape of the body - often as they are applied. For instance, tattoo artists would transfer a tattoo stencil onto the desired body location, and then iteratively tune body location, size and orientation in communication with the client. If corrections are needed, they would remove and re-apply the stencil, or sketch adjustments using sterile skin scribe markers [94]. Characteristically, templates used in maternal henna are often round and symmetrical, but the mother's belly is usually not. During application, the henna artist would then adjust the design on-the-fly by correcting for the belly's size, e.g., by adjusting the ornament's symmetry to fit the belly button's position [70]. In contrast, current design and fabrication techniques for epidermal interfaces do not afford such on-body adjustments to the individual bodyscape.

In addition, a marking's function can both follow and dictate its body location. Many types of body markings serve functions 
reaching beyond purely aesthetic or ornamental purposes. Traditional henna markings act as luck or fertility charms and are used in folk medicine [60]. In these applications, the marking's location is, for instance, determined by its healing purpose: archaeological evidence hints that ancient healing tattoos (e.g., found on 'Ötzi') overlap with acupuncture points found in Chinese medicine [13, 41] More recently, permanent 'Medical Alert Tattoos' have gained momentum, e.g., to alert first responders to chronic conditions such as diabetes $[8,42]$. Contemporary henna markings may also serve to indicate marital status [80] or as a sign of having participated in elections [30], and even for advertisement [74]. In these cases, markings are placed where good visibility and immediate discoverability can be achieved.

Similarly, the on-skin placement of modern sensing technology follows body landmarks [99]: sensors might be placed where they achieve accurate measurements and avoid stigmata, haptic actuators where tactile acuity is largest, and display elements where they are highly visible. As a result, bodyscape and circuit function form a two-way relationship, where characteristics of the body motivate choice and placement of circuit elements, and vice versa. In consequence, unlike e.g., paper circuitry design, on-body design needs not only to follow "electrical, material, and visual design principles" [47], but also adhere to design principles imposed by the user's bodyscape.

BodyStylus supports the designer in on-the-fly customization of designs, directly on the body, to fit the individual bodyscape they want to apply it to. This way, BodyStylus achieves compliance with the inherent diversity of human bodyscapes and allows matching body location to interface function.

\subsection{Mind Constraints from Geometry and Circuit Logic}

Electrical circuits contain a layer of invisible information, i.e., their inherent logic and physical rules, for instance, polarity and continuity. In consequence, circuit construction is challenging to novices, designers, and even hobbyist makers, as it involves the risk of violating electronic design rules (e.g., miswiring, short circuits) [4, 47], erroneous component selection [4], and creating functional errors [47]. When drawing circuits on skin, these issues intensify: all traces might look the same, but their functions are not. Some are essential to keep the circuit intact, some have to be isolated from each other to avoid short circuits, while others might be purely ornamental and open to artistic freedom. Combining circuit logic and artistic design increases the complexity of the circuit, making it harder to understand and debug [54]. In addition, the resistance of the circuitry can depend on the thickness of ornamental traces and on the type of conductive ink used - a characteristic which designers might find challenging [1].

BodyStylus implements free-form drawing and allows for artistic expression, but also ensures circuit logic and aesthetic qualities, such as symmetry or harmonic repetition. To achieve this, BodyStylus continuously adapts to the user's input by physically preventing mistakes that would compromise circuit logic (e.g., avoiding miswiring), or by dynamically responding to adaptations introduced by the user (e.g., change of scale).

\subsection{Use Guides for Planning and Facilitation}

There are aesthetic elements that are difficult to "get right" in freeform drawing, e.g., circular, repetitive, and evenly spaced shapes or straight lines. Many traditional on-body arts and crafts apply guides to facilitate complex shapes while finalizing and perfecting designs on the body. In tattoo art, the creation of stencils is considered an art on its own [94, 100]. While some freestyle tattoo artists despise the use of stencils as amateurish [14, 84, 94], thermal transfer stencils are today widely used to provide an outline for the tattoo artists to fill in. Similarly, off-the-shelf templates are rarely used by experienced henna artists, who instead rely on skill and muscle memory. Nevertheless, guiding tools and techniques also exist in henna art: for large henna ornaments, where spatial division is crucial, motifs are typically planned by "dotting" or outlining large elements first, and filling in details later. Guidance grids can ensure even spacing [11], and imprints from cookie cutters facilitate the free-hand application of round or symmetric shapes [79].

Stencils as guidance tools have limitations. They negatively impact the crispness of lines in make-up art and can be distorted when applied to cylindrical or convex body shapes. In addition, some guidance techniques are not directly applicable to skin. For instance, henna artists would typically practice shapes and patterning techniques on an acrylic sheet with a template underneath. When later creating henna art on skin, the artist can recall the practiced shapes or techniques from muscle memory without requiring additional guidance. This highlights how guides are useful in the learning process, specifically in skill-building activities, which has also been shown by prior work in HCI [82]. In addition, some types of guides (e.g., plastic henna stencils) confine the skin patch where ink or paste can be applied to. For instance, by creating a physical barrier. Yet, the use of more restricting or proactive guides, while known in HCI (e.g., for error prevention as in [111]), is rare in traditional crafts and underexplored in the area of on-body art.

BodyStylus enables temporary sketching of circuits, patterns or shapes, and allows using guidance marks. The users can iterate upon these virtual sketches. Once they are satisfied with their sketch, they can switch to inking mode and elaborate on the actual circuit re-using the sketch as guidance and applying constraints where needed.

\section{BODYSTYLUS}

BodyStylus supports designing and implementing epidermal interfaces directly on the body. BodyStylus consists of a general body-art inspired concept, a system including a handheld marker (Figure 1a), which enables on-the-fly design, and a set of design techniques. We now present the implementation of the BodyStylus system and introduce projected guidance and dynamic constraints as two core features to assist the user in creating epidermal interfaces that are functional, aesthetic, and customized for an individual's body.

\subsection{Implementation}

BodyStylus. shown in Figure 2a and 2b, uses a position-aware handheld pen that can proactively switch between different modes inking and non-inking - and that supports the fabrication of both conductive and non-conductive traces. The choice of a handheld 
inking device builds upon tools used in traditional body art. It allows for rich practices of designing and fabricating on and with the body, individually or collaboratively as a social activity.

Position Tracking. As a rapid means of prototyping highresolution position tracking, we opted for using a smart pen relying on printed patterns [62]. For this purpose, the current prototype is based on a Neo SmartPen M1 [86].

Tattoo Substrate. A temporary tattoo patch insulates the electrically functional parts from the body and provides the smart-pen with a trackable pattern. The pattern is laser printed onto a patch of temporary tattoo paper [61]. The tattoo paper is covered with a layer of impasto gel [18] $(\sim 0.15 \mathrm{~mm})$. The gel prevents excessive distortion of the trackable pattern by slightly evening-out the skin surface, while preserving the ability to conform to the shape of the body. The finished substrate is then reversed and adheres to the body using tattoo adhesive. The gel also has the secondary benefit of providing structural strength to the epidermal device, allowing it to be removed and re-applied multiple times. This substrate is a pragmatic choice to support the current hardware setup. In the future, systems building upon BodyStylus might use different substrates or operate directly on skin, e.g., using a different position tracking technology and an insulating spray primer.

Modular Ink Cartridges. A 3D-printed housing was attached to the Neo SmartPen (Figure 2b). The housing fit the Rotring isograph pen tip and ink-reservoir [28] to enable swappable cartridges. Cartridges with diverse non-conductive inks are available off-the-shelf, while empty cartridges can be filled with conductive ink. We use sintering-free nanoparticle gold ink [76] ( 352 $\Omega / \mathrm{cm}$ for $2 \mathrm{~mm}$ wide trace on our substrate). Pen tips of different sizes allow for controlling the thickness of ornamental and functional traces (Figure 2a). We created a customized version of the housing to hold a commercially available conductive silver ink pen [83]. Due to its aesthetic appearance we used gold ink as our default, however, for application scenarios requiring highly conductive traces, we used silver ink ( $\sim 13 \Omega / \mathrm{cm}$ for $1 \mathrm{~mm}$ wide trace on our substrate) or a combination of gold and silver inks. To increase the conductivity of functional traces we applied a primer spray that has proven successful to improve ink adhesion [68] on the painted traces.

Constraints Through Retractable Inking Tip. To prevent invalid electrical connections, the pen features a computer-controlled

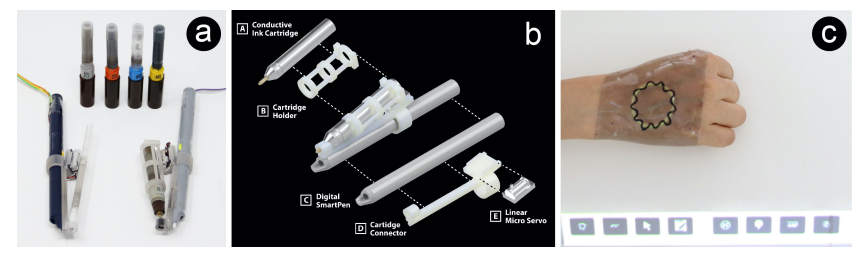

Figure 2: The handheld device includes a smart pen, multiple pen tip sizes, refillable cartridge with conductive or nonconductive ink or conductive ballpoint pen, and a servo motor. (a-b) The motor can retract the ink pen to prevent inking. Extended functionalities are accessible on the user interface menu (c). retraction system. The modular cartridge is connected to the pen via a linear servo motor [2], controlled by an Arduino Uno microcontroller. This allows for computer-operated lowering or retraction of the inking tip (Figure 2a): touching on the body, the pen delivers ink, whereas when retracted, it enters non-inking mode.

Projected In-situ Guidance. BodyStylus assists the user in fabricating epidermal devices by displaying visual cues directly on the body. Our prototype uses a ceiling-mounted projector (Sony VPL-HW50ES) for a stationary setup. A portable projector (DELL M115HD) is used for displaying guides on body locations that cannot receive projections well from the ceiling, such as the upper arm or shoulder. A simple calibration routine is implemented by marking four separate points on the substrate corners and then using projection mapping on these points. This helps the user to keep the body in the correct position during the design and fabrication process. Alternative options for future implementations include visual augmented reality, e.g., realized using a head-mounted display, or a pico projector built into the pen [87].

BodyStylus User Interface. In addition, BodyStylus includes a user interface that establishes a logic link between all of the above components. It comprises all system logic (e.g., switching between inking and non-inking mode), controls the projected visual guidance, and displays additional visual feedback and controls to the user. While most interactions are performed directly on the body, some extended functionalities (e.g., selecting electronic components and advanced visual styles) are accessible using projected buttons (Figure 2c). The Neo SmartPen sends position updates to a $\mathrm{C} \#$ host application using Bluetooth. The C\# application uses OSC to pass coordinates to the BodyStylus UI, implemented with JavaFX. The UI communicates with an Arduino microcontroller using the Arduino-serial-connector library to control the servo-motor which controls the inking tip.

\subsection{Assistance Through Guidance and Constraints}

BodyStylus considers three high-level design parameters: the aesthetics of visual patterns, the bodyscape of the user, and circuit logic. This creates a demanding design space. Users who are experts in all three areas may create a functional and aesthetic on-body circuit by free, unassisted drawing with a conductive pen. However, most users will need assistance in at least one, or multiple areas. BodyStylus combines the ease, directness, flexibility, and creative power of freely drawing on the body with various levels of assistance through two key concepts that underlie our design: guidance and constraints.

Guidance. BodyStylus guides the user with visual cues projected on the body. Guides assist the user in getting the logic of the circuit right. E.g., while the user is drawing a conductive trace, guides may suggest a routing or may highlight terminals to connect to. Similarly, aesthetic elements might be difficult to get right in the free-form drawing, e.g., repetitive or evenly spaced shapes, as well as detailed ornamental features might be difficult to draw consistently. Here, visual cues are used to guide the user in optimizing their free-hand work. Furthermore, guides help the user in choosing valid body 

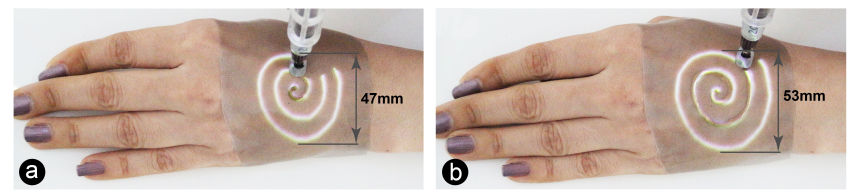

Figure 3: Visual Guidance. (a) In this example, visual cues assist the user in creating a spiral motif. (b) Guides adapt in real time to user input. As the user deviates from the proposed trajectory, the design is continuously updated.

locations for components that depend on a specific positioning on the body.

To support improvisation during the inking process, guides can be set to adapt continuously while the user is sketching (see Figure 3 ). Hence the user can decide at any point to follow a design suggestion or not. In the latter case, the visual guide is updated to be in line with the modified design.

The level of detail of guides can be adjusted to adapt to the user's expertise and the desired degree of creative design freedom. For instance, visual guides can simply project outlines or directional indicators but if desired can include full details of a design, e.g., the detailed routing of a trace or the full visual pattern of an ornament.

Constraints. To prevent the user from inadvertently violating constraints relating to circuit logic, aesthetics, or body location, the pen can automatically switch between an inking mode and a non-inking mode. This enables the system to physically restrict the user's inking actions. For instance, when the user is about to create a short-circuit, the pen can automatically stop inking by moving the tip of the cartridge up.

To free up creativity but provide safe boundaries for flexible exploration, the system allows the user to suspend constraints with a "manual override" (c.f., Zoran et al. [111]). In this case, the user repeats the desired action a second time to override the constraint.

Despite the pen only offering two distinct interactive modes (inking and non-inking), we define the constraint space as a continuum that allows more or less tolerance. At the strictest, it prevents any inking that was not planned out by the initial design (e.g., deviating from an original aesthetic). At the lowest level, it does

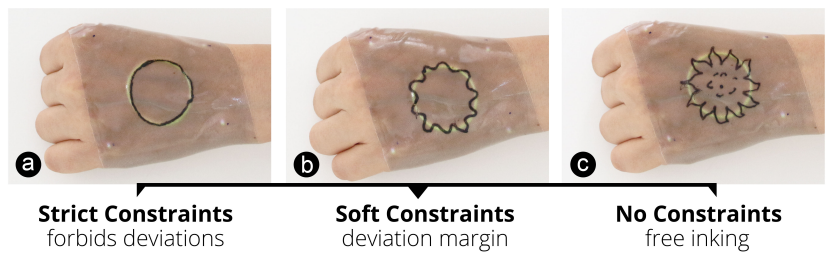

Figure 4: Constraints can be imposed at different levels. In this example, a strict constraint forces the user to closely follow the projected shape primitive (yellow circle, a). A soft constraint allows the user to deviate within a certain margin, for creative adjustments (b). Without constraints imposed, the user can draw freely, while still benefiting from highlevel visual guidance (c).

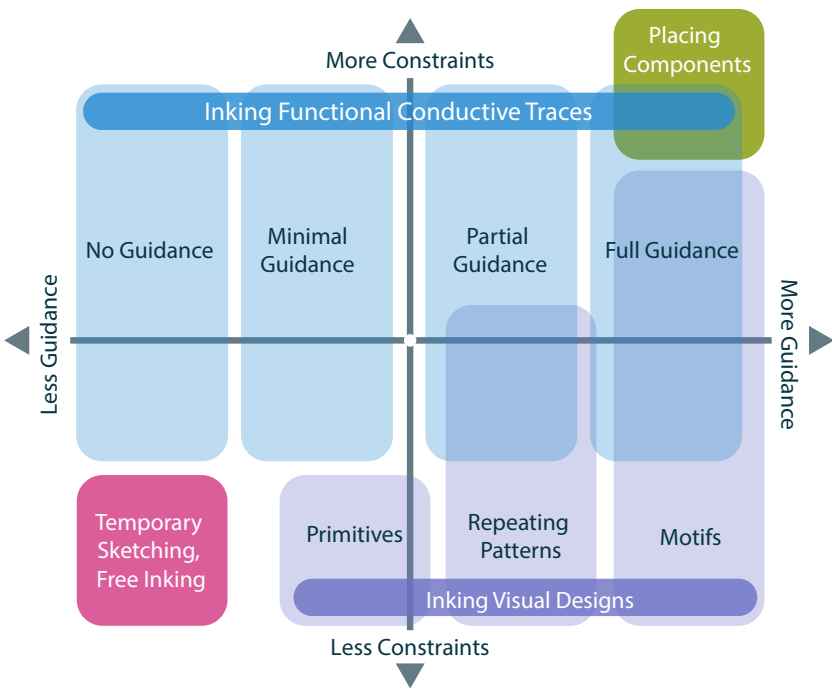

Figure 5: Guidance ( $x$-axis) and constraints ( $y$-axis) are orthogonal and complement each other during the fabrication process. While crafting a complete epidermal device, a user might flexibly move within this two-dimensional space depending on the current task. Free-form components combine design methods from inking conductive traces (blue), placing components (green) and inking visual designs (purple).

not restrict the user's actions at all. Between the two extremes, it allows deviations within a certain margin, as defined by the user (see Figure 4).

Guidance and constraints can be flexibly combined throughout the design and fabrication process. This creates a two-dimensional space for interacting with BodyStylus, visualized in Figure 5. Depending on the current design task and her expertise, the user may benefit from more or less guidance and more or less constraints, for working on circuit logic, aesthetics, and bodyscape.

\section{DESIGN AND FABRICATION TECHNIQUES}

We now present a set of interaction techniques for on-body design and fabrication with BodyStylus and describe how these are shaped by constraints and guidance (see also Figure 5).

\subsection{Temporary Sketching and Free, Permanent Inking}

Before any part of the design becomes permanent, one can use the pen to draw virtual lines in the non-inking mode. These might be used to sketch an outline of the design or to experiment with how lines might complement the shapes of the body. As these virtual lines are not physically inked but merely indicated using visual feedback, users can move, scale, delete, and redraw as they wish. If one is certain of the pattern one wishes to place on the body, BodyStylus supports drawing of conductive and non-conductive traces by switching to the inking mode. 

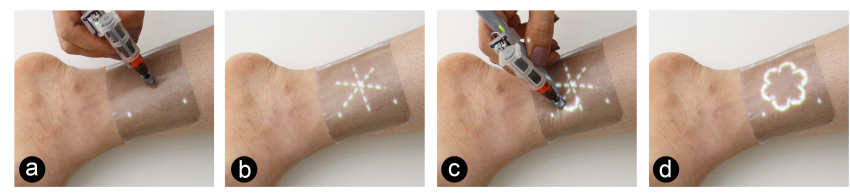

Figure 6: Radial symmetry tool enabling rapid designs of repeating patterns, building on practices from henna art. The user positions the center of the symmetry axes (a), these axes appear as guides (b), the user draws the geometrical pattern in one sector (c), the pattern is replicated in the other sectors (d).

\subsection{Inking Visual Designs}

In inking mode, BodyStylus offers primitives as visual guides for artists to develop their designs, patterning tools to support artists in creating repeating patterns and motifs for adding specific predefined elements to the design.

Shape primitives such as line, circle, and spiral provide guidance to orient or outline the design. For instance, the user can select the circle tool, then specify its center and radius with a stroke to use it as a guide to draw a flower.

Repeating patterns are an important characteristic of henna art, which we draw on for aesthetic inspiration. We provide visual guidance for creating multi-axial symmetries, repetitions of patterns, and fractal patterns. As an example, to draw a complex pattern with radial symmetry, the user selects the radial symmetry tool and locates the center point on the canvas (Figure 6a). The user selects the number of radial axes and (Figure $6 \mathrm{~b}$ ) sketches the desired design in one of the sectors (Figure $6 \mathrm{c}$ ). The pattern is then projected in all other sectors for the user to trace (Figure 6d).

Finally, the user can select to transfer motifs to the body. The user has the option to select one of the available patterns (e.g., horseshoe or curlicue) or upload an SVG line graphic. This shape is then projected on the body at a location specified by the user. Here the system expects the user to trace the projected lines. If the user deviates from the projected line, the system scales and rotates the shape to allow the user to dynamically adjust the shape through drawing (Figure 3). Constraints can be used to limit the amount the user can deviate from the predefined shape.

Here, guidance is consecutively increased. Primitives are merely used as helper lines and remain unaffected by the user once placed. Tools for repeating patterns allow the user to create detailed templates - the system assumes that the user will trace this, but it is not enforced. Finally, motifs assume the user will always trace them, even dynamically updating to adjust to the user. In the dimensionspace on Figure 5, we move from left primitives gradually further right repeating patterns and further up for motifs.

\subsection{Inking Functional Conductive Traces}

BodyStylus is designed to support inking electrically functional designs. In doing so, the user should be supported to create valid circuits while at the same time being free to creatively adjust the design to their desired visual aesthetics. For instance, instead of drawing the most efficient connection between two components,
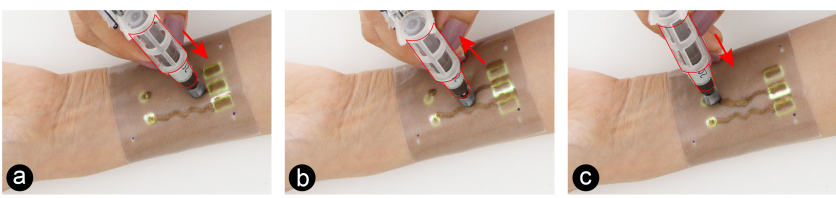

Figure 7: Constraints prevent invalid connections of conductive traces. The user draws a conductive trace to connect two terminals (a). As she is about to connect to another conductive trace and create a short-circuit, the pen retracts to noninking mode (b). Moving the pen away from the original trace switches the pen back to inking mode (c).

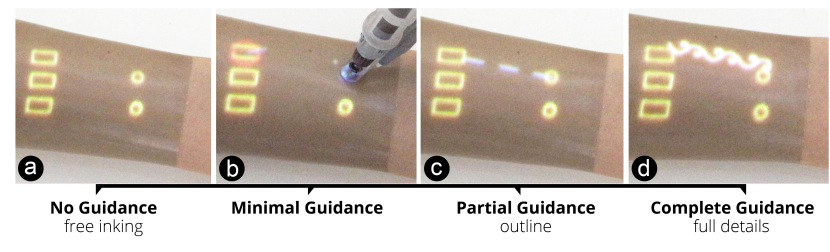

Figure 8: Varying levels of guidance to draw the conductive trace between two terminals. Without guidance, the user must draw freely and connect the correct pins (a). Minimal guidance highlights other pads the user can connect to (b). With partial guidance, the outline of the trace is displayed as a guide to route the connection (c). Full guidance shows a detailed pattern of the trace $(d)$.

users might deliberately deviate to create a more aesthetically pleasing result, or augment a trace with artistic elements, e.g., curlicues.

Again, the interaction methods are based around an interplay of guidance, which shows the users which traces or pads can connect, and constraints, which physically prevent the user from creating invalid connections (short circuits, wrong polarity - see also Figure 7). To achieve this, the system requires a model of the underlying electrical circuit; BodyStylus records the precise location of the pen on the body while inking and saves this data as a vector graphic. In addition, the system tracks the position and polarity of the embedded components and distinguishes between functional and artistic traces.

For working with electrically functional traces, BodyStylus supports four levels of guidance (Figure 8). To work completely freely, users might choose no guidance (Figure 8a). To add some visual support minimal guidance can be chosen to highlight which other pads the current trace can connect to (Figure 8b). If using partial guidance, a direct path to a suggested destination pad is highlighted (Figure 8c, dashed line). Finally, complete guidance shows a path the user needs to trace to complete the circuit. This path might include basic routing (using the $\mathrm{A}^{*}$ algorithm [25]) and can include visual styles for aesthetic effects (Figure $8 \mathrm{~d}$ ). The desired style is manually chosen from a library of patterns. The above guidance methods were described from left to right of the dimension space in Figure 5. They can be crossed with continuously varying levels of constraints, which change the margin of how close one may come 
to making an error before the pen retracts, moving up or down in Figure 5.

\subsection{Placing Electronic Components}

To assist users in adding standard electronic components to the epidermal circuits, BodyStylus contains a library of frequently used components. Our current implementation comprises resistors, capacitors, diodes, LEDs, inductors, and breakouts for connecting cables. Additional components can be implemented: for each component, the library stores the package type and function of pins.

Using this information, BodyStylus visually guides the user in placing the component. After selecting a component from the library, the user defines the location of the first pad (Figure 9a) and drags the pen to locate the last pad. The system guides the user by projecting the distance and angle between pads (Figure 9b). After releasing the pen, the system displays the component's footprints (Figure 9c). To make the design permanent, the user starts inking the pads. The system constrains painting outside the footprints' borders and connection between terminals by retracting the pen (Figure 9c). Finally, the user places the components (e.g., LED) and connects the wires to the terminals using conductive paste as glue [10] (Figure 9d). Due to the requirements for strict constraints, these interactions are to the top of the dimension space in Figure 5.

\subsection{Free-Form Components}

Some commonly used types of electronic components can be handdrawn in free -form using conductive ink, e.g., capacitive touch buttons, sliders, resistive bend sensors, strain gauges, or coils. These provide two opportunities: (a) the user can adjust their function to meet the specific demand of the system (number of windings in coil, length of bend sensor, number of pads on a slider, etc.), and (b) the user can adapt their aesthetics to match the overall design.

To provide support for these activities, BodyStylus offers a library of parametric designs for free-form components (resistive bend sensors, capacitive sliders, and coils; additional components can be added, similarly to electrical components). To create a free-form component, the user selects a parametric design and places it on the body (Figure 10a). The design can be moved, scaled, and adjusted in visual style (Figure 10b). If body placement constraints are violated (e.g., placing a bend sensor at a location that cannot bend), the component is highlighted in red color (Figure 10c). When satisfied, the user can ink the traces with conductive ink. Of note, the user is free to creatively deviate from the design and to add ornaments that
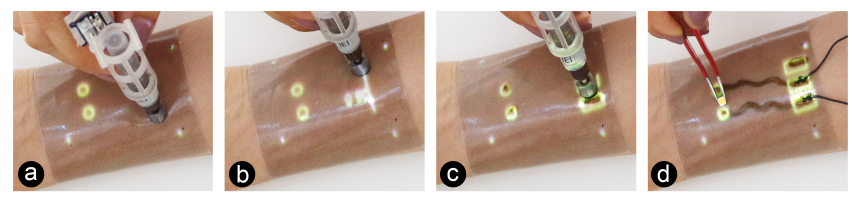

Figure 9: Placing electronic components. The user defines the location of a component's pad (a) then drags the pen to define the distance and orientation of other pads (b). The system displays the footprints and the user starts inking (c). Finally, the user connects components using bare conductive (d).

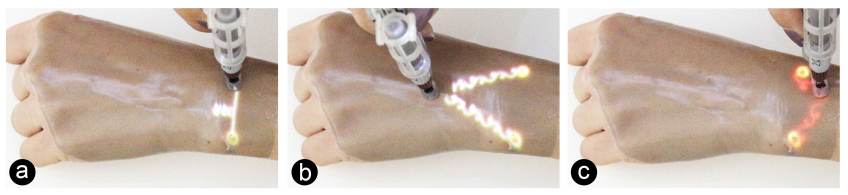

Figure 10: Parametric components. The user places a bend sensor design on the wrist (a) and enhances its visual aesthetic with a curlicue pattern. She drags the center, which modifies the pattern on-the-fly (b). When moving the sensor to a body location the user cannot bend, the body constraint is highlighted in red (c).

match the aesthetics of the overall design. Figure 11 shows various examples of how the user can flexibly adapt the basic parametric design to create different aesthetics. Of course, a specific aesthetic style can be encapsulated as part of a parametric design, too. For instance, the slider component allows the user to choose from several visual styles for the conductive line.

Creating parametric components borrows from previously introduced interaction methods: terminals are implemented as electrical components, connections within the free-from component are implemented as described for electrical traces, and specific shape features are implemented as described for aesthetic patterns. Of course one can combine these with inked visual designs (see also Figure 9), and temporary sketching can be used for quickly testing ideas before permanently implementing them.

\section{EVALUATION}

To validate the practical feasibility and to investigate opportunities and limitations of the BodyStylus concept, workflow, and example implementation, we present (a) examples of implemented designs alongside lessons learned, including a detailed walkthrough of how one of these designs was created and (b) feedback from a focusgroup exploration with three stage make-up artist trainees and a focus group exploration with three engineers.

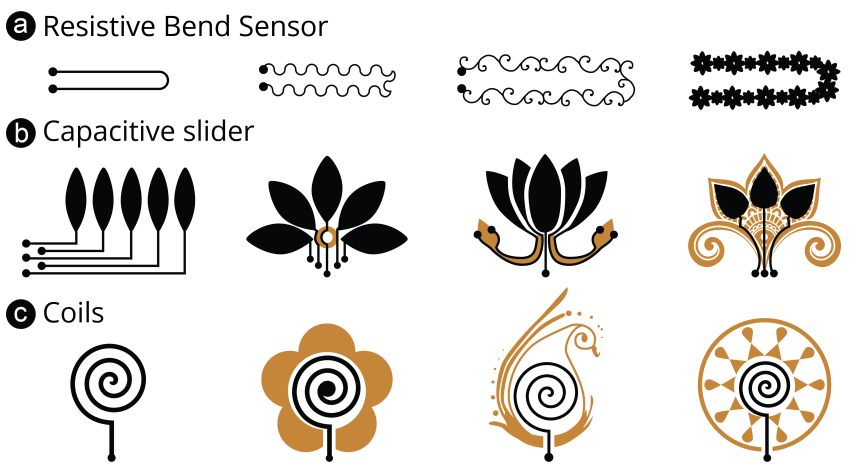

Figure 11: Custom free-form components can be created by selecting a parametric design (leftmost column) and then customizing it in size and style. 


\subsection{Application Cases Realized with BodyStylus}

To demonstrate the end-to-end feasibility of epidermal interfaces, we present four example application cases we have designed and fabricated with BodyStylus: an interactive hand adornment, a wirelessly powered epidermal interface, a wristband control, and a decorative anklet. The functional prototypes are shown in Figure 12.

6.1.1 Interactive Hand Decoration. Inspired by interactive makeup such as Kinisi [91] and traditional bridal henna [60], we decided to create an interactive hand adornment that reacts to hand movement with light (Figure 12a). To describe the design and fabrication process, we present a step-by-step walk-through of this application case before presenting the three other application cases more briefly.

An important part of practicing body-arts is the preparation of the body, for example, a make-up artist applies a coat of primer to create a blank slate. Similarly, the empty substrate provides a blank slate for drawing on, and its placement already foreshadows its potential use. In this case, we placed the empty tattoo on the hand and wrist to support the implementation of a bend-sensor and hand adornments.

Henna artists usually start their work by "placing dots" as guidance for subsequent patterns. We decided to start with the bend sensor and, similarly, initially placed control points where other parts of the circuit will later connect to. Once satisfied with the placement, the terminals were made permanent by inking them and choosing a visual style for the sensor (Figures 13a and 13b).

Artists consider the body's movements as an important reason for working on the body, to see the movement of the person and to see the response of the materials. Similarly, designing the bend sensor directly on the body allowed for exploring where exactly the wrist moves and deciding how the electrical traces cover this movement (Figure 13c). The position and size of the sensor relative to where movement occurs both influence the function of the design
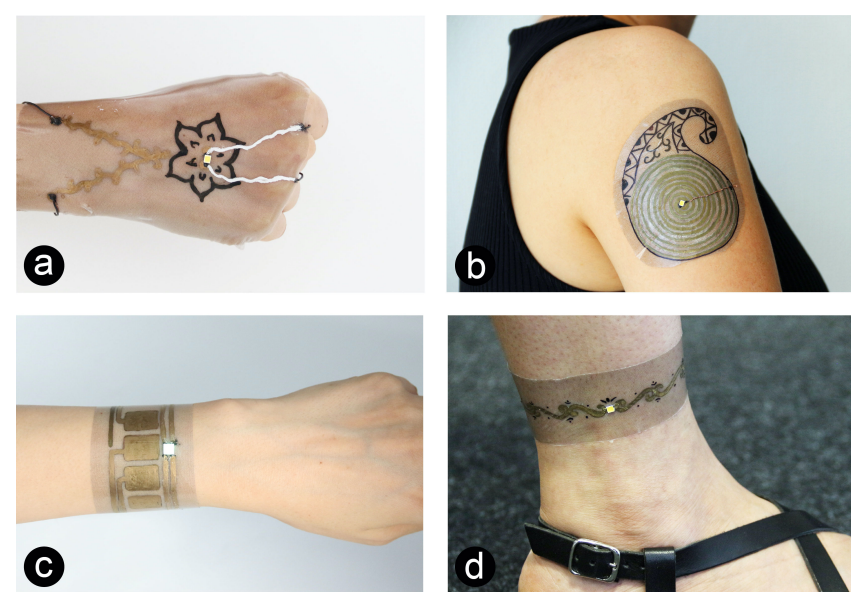

Figure 12: Interactive Application Cases. Interactive hand decoration that responds to body movement (a); wirelessly powered interface (b); wristband slider control (c); decorative anklet (d).
At the same time, the pattern selected for the sensor shapes the design's aesthetics. For now, we did not ink the traces so we could return to tweak and fine-tune them later.

Next we started sketching a flower design. As its center will be an LED, we first defined its footprint (Figure 13d). Then, we started sketching its adornments. Here the use of the radial symmetry tool was again reminiscent of traditional henna practice, e.g. drawing a crosshair as guidance. Similarly, we defined a 6-segment crosshair (Figure 13e), which we used to create a repeating pattern (Figure 13f). We then proceeded to immediately ink it using nonconductive ink (Figure 13g). Satisfied with our arrangement of the flower and sensor (Figure 13h), we also inked the sensor using gold ink as well as the connectors to the LED with conductive silver ink, using free inking without guidance (Figure 13i). In case of an error, BodyStylus would prevent us from creating a short-circuit or from inadvertently connecting to the bend sensor.

In traditional henna practice, once bridal henna has dyed the skin and the paste is removed, it is not uncommon to add gemstones or other adornments, which are quite literally glued to the skin. Much in the same way, we finalized our design by placing the LED (Figure 13j) as well as connecting our prepared traces to an Arduino micro-controller. The finished piece can be seen in Figure 13k. When the wrist is flexed, the resistance in the bend sensor increases and the LED is turned on (Figure 13l).

6.1.2 Wirelessly Powered Epidermal Interface: Inspired by [32], we set out to design and fabricate an epidermal interface for use on one's upper arm that is wirelessly powered (Figure 12b). It features an LED embedded inside an aesthetic line-art design. When powered using an off-the-shelf wireless charging coil [50] held in close distance to the interface, the LED lights up. We selected the Coil free-form component and used visual guidance for creating a spiral that acts as a receiver coil for inductive power transmission. We
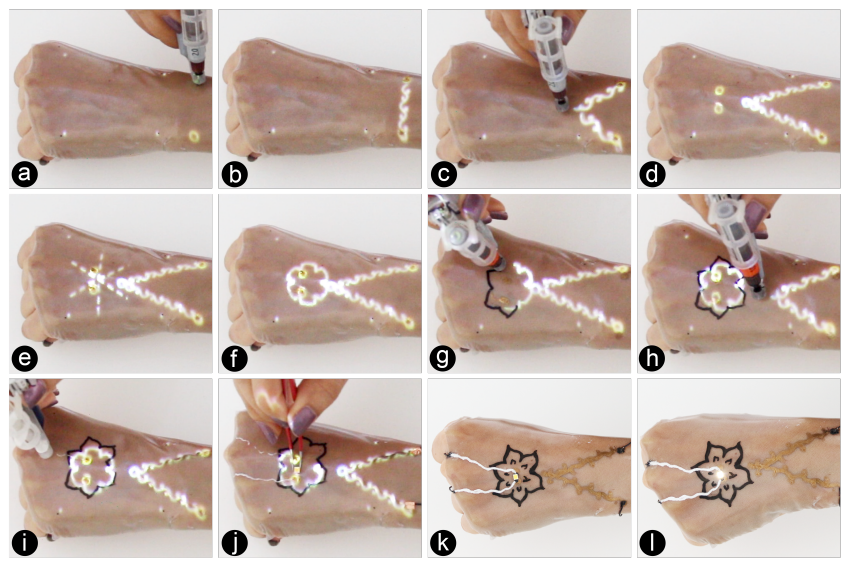

Figure 13: Design and fabrication steps of Shape Responsive Hand Decoration. BodyStylus supports the design and fabrication of visually aesthetic epidermal devices. The system guides the user by projecting the electronics, aesthetics, and body constraints and prevent logic error (constraints) by retracting the ink cartridge. The patterns are designed using conductive gold ink, silver pen, and regular black ink. 
used conductive yarn with a non-conductive coating to close the coil. As the conductivity of the conductive gold ink is not high enough for creating an induction coil, we realized the spiral with silver ink. For a visual style similar to henna dye, we then repeated the trace with gold ink. To make the design aesthetically pleasant, we experimented with several ornamental shapes and decided to surround the spiral with a paisley design, a key element of henna art, using free inking.

While creating the design, we realized that the use of impasto gel underneath the temporary tattoo substrate allows the substrate to be removed and re-applied multiple times. This creates opportunities for combining the benefits of on-body design with working off-body for tasks such as finishing complex patterns or debugging. The design has been done collaboratively on the body and fitted to the size and dimension of the user's arm, and then finalized on a table before it was transferred again to the body.

6.1.3 Wristband Slider Control: In our third application case, we aimed to investigate capacitive sensing. We have realized a wristband that comprises a capacitive slider with three segments and an RGB LED (Figure 12c). We selected the slider free-form component and used visual guidance to design and fit the size and number of segments to the wrist. The full constraint prevented us to connect the segments to each other inadvertently. Using projected guidance, we could design several sliders with different sizes, shapes, and number of segments at different angles to find the most suitable design without investing time to fabricate them. The device was created by a single user on her own body, and then connected and controlled with an Arduino microcontroller. In our proof-ofconcept, the capacitive slider is used to adjust the color of the LED; in future implementations, a wireless data connection could be easily added to turn the device into a generic body-based controller, e.g. for controlling presentation slides, a music player, room lighting, amongst other options.

6.1.4 Decorative Anklet: To demonstrate that BodyStylus is applicable to various body locations, we realized a decorative anklet that features a blinking LED (Figure 12d). The LED footprint was selected from the component library and then placed in the desired position on the ankle. We used full detailed guidance to project the curlicue patterns between LED and the breakout footprint. The pattern was painted with gold ink and then connected to an SMD LED using bare conductive ink. An Arduino microcontroller is used to power and control the device.

\subsection{Focus Group Explorations}

We conducted two hands-on explorations to collect feedback of BodyStylus in use, following an approach that has proven valuable to connect with craft experts [7].

6.2.1 Method. The first exploration was conducted with three engineers familiar with designing circuitry, having backgrounds in embedded systems (E1, female, 24), robotics (E2, male, 28), and physiological sensing (E3, male, 30). The second group consisted of three professional stage make-up artists, all female, aged 26 (A1), 22 (A2), and 19 (A3), and experienced with a wide repertoire of onbody techniques, including the use of different mediums, pigments, and tools.
Before the exploration, we ensured that all participants had a working understanding of what epidermal devices are. After providing informed consent, both groups were asked to perform a series of tasks designed to elicit discussion and feedback. The sequence of tasks was arranged from simple to more complex, to allow participants to become familiar with BodyStylus. They were asked to (1) draw two intersecting lines on a mannequin, using the projection for guidance, and automatic retraction for constraints; (2) create a freehand pattern including one interface element (e.g., a button) on one of their colleagues; and (3) collaboratively design and implement a functional prototype. For the engineers, this was a generic controller, while the make-up artists were tasked to build a device for controlling RGB LEDs as used in a design they were working on at their theatre. We paused after each task, inviting critique and probing for observations and introspections. Sessions were audiorecorded, and photos of key situations were taken. Claims about the system, applications, or usage were extracted into individual files, reducing the 4 hours of recording to 33 minutes of tagged quotes. These quotes were thematically grouped as reported below.

6.2.2 Results \& Discussion. Both groups were able to complete all tasks and provided valuable feedback. Most critically, after less than two hours of exploration, they were able to implement their own, functional, epidermal UI without intervention from our side. We now summarize central findings by theme.

BodyStylus for Bodily Exploration. Working on other people came naturally to the make-up artists, who do so in their day-today practice, while the engineers tended to show short moments of hesitation before inking each other. However, the engineers took advantage of working on the body rather than in CAD software, for example, for quickly testing the positioning of UI elements on moving body parts (Figure 14b).

For task two we placed tattoo substrate on the knuckles to see how participants might integrate these complex shapes into their designs. After asking participants to create input elements, we observed different ways in which designs reacted to body features: E1 lined up her interface elements with the knuckles, while E2 superimposed a button on a knuckle. E3 chose a different approach: He drew a strain gauge over the knuckles. Make-up artists engaged with the body differently for their designs. Instead of physiology they focused primarily on the visibility of body parts. In the first design they created for task 2, A3 placed the button as far to the edge of the substrate as possible, with the intent of hiding the interface. Latter designs by A1 and A2 explored opportunities of using the button as an element visible to others.

Task three showed that the real-world application of the makeup artists was valuable in critically reflecting on design. While the engineers merely placed buttons on the arm (see Figure 14c), the make-up artists spent more time discussing and planning their final interface. They highlighted aspects such as benefits of unimanual vs. bi-manual operation, discussed which surfaces were better suited for pressure and which for touch input, and planned on how to avoid unintentional activation. Working on the body led the make-up artists not only to think about the visual layout of the interface, but also reflect on how the epidermal UI will interact with the body when in use. 


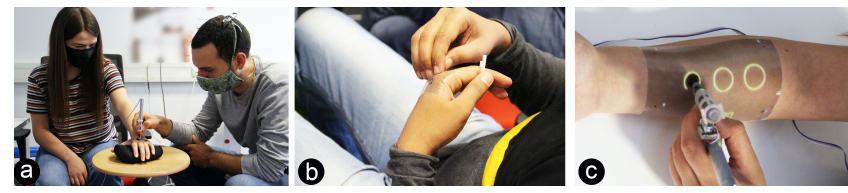

Figure 14: Engineers during focus group exploration. Inking each other (a), testing imagined UI elements (b), using guidance for implementing generic touch-controller (c).

BodyStylus as Artistic Hand Tool. The engineers immediately praised the high ink-flow rate, which they felt made the device easy to use and promised robust circuits. Interestingly the make-up artists remarked negatively at the high ink-flow rate, and especially with the fact that the ink-flow rate could not be continuously adjusted while inking. They felt that this inhibited their ability to produce delicate patterns and dynamic stroke thickness. While all participants were able to adapt to the offset between the inking tip and tracking tip, the make-up artists repeatedly stressed that it required active attention - especially on the uneven and compliant surfaces of the body. Interestingly this too was less of an issue for the engineers.

While we anticipated that participants might be apprehensive working with an actuated tool that could retract based on predetermined rules, this was largely not the case. In addition, we did not observe any effect on the user's drawing while the pen was retracting. E1 mentioned that she felt the pen should be designed to minimize recoil when the inking tip was retracted. A2 explained that because she understands the behavior of BodyStylus, it is just a tool, much like the various pens and brushes she uses in her everyday practice.

BodyStylus as Error Prevention. The engineers commented on the usefulness of constraints for positioning ICs and SMD components. Beyond that, E2 and E3 were especially excited that constraints provide them with the ability to draw finely detailed components such as strain-gauges. E2 felt that such constraints might be a useful proxy for physiological sensing, for example in place of EMG, while E3 stated that he would enjoy inking with constraints on other surfaces than the body: "whenever I build something with elastic materials and want to measure deformation, I'd love to use this for drawing sensors". For the make-up artists, the use of constraints for error prevention was mostly appreciated in the context of tasks requiring precise positioning and exact stroke lengths: "oh, I want this next time I do eyes" (A3). Nevertheless, it

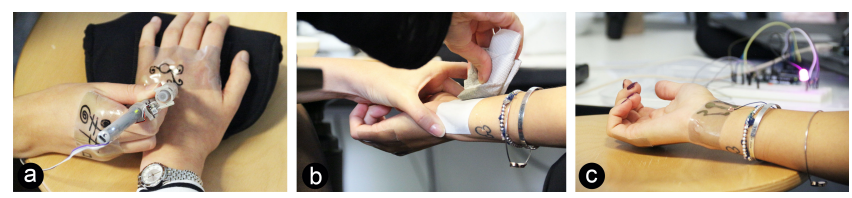

Figure 15: Artists during focus group exploration. Inking on each-other (a), applying substrate for functional prototype interface (b), completed epidermal interface controlling an RGB LED (c). was also noted that constraints were only applicable to the very specific context of their craft, as in make-up one usually works with smooth gradients. Participants also expressed worry that overreliance on the system might risk losing their manual skills. Along these lines, A3 stressed the importance of being able to override constraints.

BodyStylus as Assisting Guide. With the simple examples created during the exploration, the engineers felt that they did not need the guidance features. They did speculate that it would be useful for someone with less knowledge of electronics. The make-up artists in fact did appreciate the guidance. For instance, A3 noted that the projected guides would blend in with make-up techniques making use of the human face's symmetry: "there are these lines" [she points from nostril to outer edge of eye-socket] "which we use, and if you could project them, I would immediately use that". The guides blend in with their existing craft. For example, A1 mentioned that "we already sometimes draw dots to guide the design" and "having this would be so much better". All three artists stated they would want to use guidance in their everyday practice.

BodyStylus Integrated in Day-to-Day Practice. Interestingly, the artists' take on how the system should be used changed while they gained more experience with it. Initially, they envisioned making use of BodyStylus for final touches or for implementing a carefully thought-out design. Towards the end of the session, they noted that BodyStylus would be most handy as a sketching tool early on in the design process. They outlined how it could enable them to rapidly create a rough on-body draft so that they would have more time to work on the final details. The engineers questioned why the system was constrained to working on the body and highlighted that they would appreciate this approach for rapid prototyping in other contexts also.

6.2.3 Summary. Participants took advantage of working directly on the body by exploring bodyscape, using the morphology of the body, and testing UI elements in-situ. Differences in expectations and goals led to conflicting assessments of the physical pen prototype. In future iterations, dynamic ink-flow should be considered, and the inking and sensing pen-tips superimposed. Both engineers and artists appreciated constraints; however, constraints are more useful to engineers, who suggested applications for constraints beyond those we anticipated. Artists worry that overrelying on constraints might prevent maintaining and developing manual skills. Inversely, guidance appeared more suitable to the artists, who this time were the ones to suggest new applications. Finally, BodyStylus not only supported complete novices in the design and fabrication of a working prototype, but also facilitated thinking about interaction and design.

\section{DISCUSSION}

Occlusion and Calibration. Our implementation of in-situ visual feedback is currently restricted to a projector, which can cause occlusion on the skin while the user is manipulating the pen. Accurate projection mapping on a moving and deforming body is still an active research field. We implemented a simple calibration step for mapping the projection to the skin site's position, which however 
does not continuously update, hence requiring the skin site to remain immobile during the interaction. We experienced that this is acceptable when designing on limbs, as they can be placed in a comfortable resting position, but can be more challenging when designing on the torso. Note that thanks to the locally stable Anoto pattern, the position tracking remains accurate in case a skin site is moving in 3D space, which is important to maintain a correct digital model and ensure precise computer-controlled inking behavior. In future work, we plan to experiment with a pico projector built into the pen [87] and a head-mounted display for improving mobility and avoiding occlusions and to implement a more advanced projection mapping scheme as suggested in [65].

Focus on Hand and Arm. According to [24], the hand/arm space is the one most extensively used for on-body interfaces. Although our main focus is on these locations, evidence from the implemented example cases confirms that our approach works well on the demonstrated body parts (e.g., hand, forearm, upper arm, wrist, and ankle). It can be extended to other body parts, such as legs, thigh, and back (the latter requiring co-creation since self-inking is not possible on the back). On very challenging skin geometries, e.g., strongly convex body parts, the smart pen sometimes loses data points; interpolating the captured data allows estimating the pen position.

Substrate. Our current setup uses a substrate printed with dot patterns to enable the smart pen's position tracking. The choice of the substrate is a pragmatic choice to support this particular proofof-concept implementation with precise tracking and an insulation layer. However, future iterations based on BodyStylus might be designed to operate more directly on skin. One option is to make the substrate fully transparent, e.g., by printing the Anoto pattern using a transparent IR absorbing ink. Another option is to remove the substrate altogether, using a different position tracking technology, e.g., optical motion capturing, combined with an insulated spray primer. In addition, the strengths of BodyStylus include its support of skill-building activities using guidance and constraints as well as its ability to be used with self-sintering, skin-safe gold ink. As a result, artists could practice designs on themselves using guidance and constraints to commit drawn shapes to muscle memory, and then, removing the substrate and using only the pen with gold ink recall the practiced shape when designing for a wearer.

Single-layer Substrate. While our approach is currently restricted to single-layer, conductive bridges can be realized with electrically insulated conductive yarn.

Modifying Traces. Lastly, while our system supports users before and during inking, it does not allow the user to modify traces after they have been made. Future work should investigate erasing interactions [59] to edit the design after inking.

\section{CONCLUSION}

Until now, design and fabrication processes for epidermal devices were indirect and detached from the human bodyscape. With BodyStylus we presented a novel interactive approach that overcomes this limitation and allows us to design and craft epidermal devices directly on the body using a hand-held fabrication tool and self-sintering conductive ink. We present a palette of on-body design and fabrication techniques that leverage in-situ projected guidance and physical constraints to facilitate the creation of functional and aesthetic epidermal devices. BodyStylus is conceptually grounded on design considerations integrating circuit logic, aesthetic principles, body shape, and functionality, and expands upon the existing traditions of body markings by facilitating skill-building activities. We exemplify these parallels and starting points using four concrete application cases, where we further demonstrate how BodyStylus succeeds in creating functional epidermal devices. Our results show that our approach was positively received by stage make-up artists and engineers with an electronics background, and succeeds in moving the design and fabrication process from computer and workbench on to the body, making it more direct and immediate.

\section{ACKNOWLEDGMENTS}

This project received funding from the European Research Council (ERC) under the European Union's Horizon 2020 research and innovation program (grant agreement No 714797, StG Interactive Skin). We thank Martin Weigel and Anusha Withana for their valuable feedback, Muhammad Hamid for his help with video editing and Robert Strahl for his support in gold ink sample preparation.

\section{REFERENCES}

[1] LED Henna Tattoos Make You Look Like a Bohemian Cyborg. 2020. https://www.vice.com/en_uk/article/53w93x/led-henna-tattoos-make-youlook-like-a-bohemian-cyborg. Accessed: 2020-04-01.

[2] Linear Micro Servo For Ultra-Micro aircraft. 2020. https://www.amazon.com/ Digital-Loading-Actuator-Ultra-Micro-Aircraft/dp/B07G34BT9N. Accessed: 2020-03-16.

[3] Joanna Bergstrom-Lehtovirta, Sebastian Boring, and Kasper Hornbæk. 2017. Placing and Recalling Virtual Items on the Skin. In Proceedings of the 2017 CHI Conference on Human Factors in Computing Systems (Denver, Colorado, USA) (CHI '17). ACM, New York, NY, USA, 1497-1507. https://doi.org/10.1145/ 3025453.3026030

[4] Tracey Booth, Simone Stumpf, Jon Bird, and Sara Jones. 2016. Crossed Wires: Investigating the Problems of End-User Developers in a Physical Computing Task. In Proceedings of the 2016 CHI Conference on Human Factors in Computing Systems (San Jose, California, USA) (CHI '16). Association for Computing Machinery, New York, NY, USA, 3485-3497. https://doi.org/10.1145/2858036.2858533

[5] Leah Buechley, Sue Hendrix, and Mike Eisenberg. 2009. Paints, Paper, and Programs: First Steps toward the Computational Sketchbook. In Proceedings of the $3 r d$ International Conference on Tangible and Embedded Interaction (Cambridge, United Kingdom) (TEI '09). Association for Computing Machinery, New York, NY, USA, 9-12. https://doi.org/10.1145/1517664.1517670

[6] Varun Perumal C and Daniel Wigdor. 2015. Printem: Instant Printed Circuit Boards with Standard Office Printers and amp; Inks. In Proceedings of the 28th Annual ACM Symposium on User Interface Software and amp; Technology (Charlotte, NC, USA) (UIST '15). Association for Computing Machinery, New York, NY, USA, 243-251. https://doi.org/10.1145/2807442.2807511

[7] Vanessa Julia Carpenter, Amanda Willis, Nikolaj “Dzl” Møbius, and Dan Overholt. 2019. Electronic Kintsugi. In Proceedings of the Future Technologies Conference (FTC) 2018, Kohei Arai, Rahul Bhatia, and Supriya Kapoor (Eds.). Springer International Publishing, Cham, 104-121.

[8] Sarah Chadwick and Mamta Shah. 2013. Tattoos: ancient body art may assist in medical emergencies. European fournal of Pediatrics 172, 7 (2013), 995-995. https://doi.org/10.1007/s00431-013-1971-1

[9] Youngkyung Choi, Neung Ryu, Myung Jin Kim, Artem Dementyev, and Andrea Bianchi. 2020. BodyPrinter: Fabricating Circuits Directly on the Skin at Arbitrary Locations Using a Wearable Compact Plotter. In Proceedings of the 33rd Annual ACM Symposium on User Interface Software and Technology (Virtual Event, USA) (UIST '20). Association for Computing Machinery, New York, NY, USA, 554-564. https://doi.org/10.1145/3379337.3415840

[10] Bare Conductive. 2020. https://www.bareconductive.com. Accessed: 2020-02-15.

[11] Nadine Dafrawy. 2019. 5 Complicated Geometric Henna Designs Completely Simplified (A Step-By-Step Guide). https://www.thehennaproject.net/post/ geometrichennadesigns Accessed: 2020-09-01.

[12] Ella Dagan, Elena Márquez Segura, Ferran Altarriba Bertran, Miguel Flores, Robb Mitchell, and Katherine Isbister. 2019. Design Framework for Social Wearables. In Proceedings of the 2019 on Designing Interactive Systems Conference (San Diego, 
CA, USA) (DIS '19). Association for Computing Machinery, New York, NY, USA 1001-1015. https://doi.org/10.1145/3322276.3322291

[13] Leopold Dorfer, Maximilian Moser, Frank Bahr, Konrad Spindler, Eduard EgarterVigl, Sonia Giullén, Gottfried Dohr, and Thomas Kenner. 1999. A medical report from the stone age? The Lancet 354, 9183 (1999), 1023-1025.

[14] C.W. Eldridge. 2004. The Tattoo Archive - Stencils. https://www.tattooarchive com/history/stencils.php

[15] L Furey. 2017. Archaeological evidence for tattooing in Polynesia and Micronesia In Ancient Ink: The archaeology of tattooing, Lars Krutak and Aaron DeterWolf (Eds.). University of Washington Press, Washington, Chapter 11, 159-184. http://www.jstor.org/stable/j.ctvcwnc53

[16] Madeline Gannon, Tovi Grossman, and George Fitzmaurice. 2015. Tactum: A Skin-Centric Approach to Digital Design and Fabrication. In Proceedings of the 33rd Annual ACM Conference on Human Factors in Computing Systems (Seoul, Republic of Korea) (CHI '15). ACM, New York, NY, USA, 1779-1788. https://doi.org/10.1145/2702123.2702581

[17] Madeline Gannon, Tovi Grossman, and George Fitzmaurice. 2016. ExoSkin: OnBody Fabrication. In Proceedings of the 2016 CHI Conference on Human Factors in Computing Systems (San Jose, California, USA) (CHI '16). ACM, New York, NY, USA, 5996-6007. https://doi.org/10.1145/2858036.2858576

[18] Impasto gel. 2020. https://lascaux.ch/en/products/painting-mediums/lascauximpasto-gels. Accessed: 2020-09-06.

[19] Daniel Groeger and Jürgen Steimle. 2019. LASEC: Instant Fabrication of Stretchable Circuits Using a Laser Cutter. In Proceedings of the $2019 \mathrm{CH}$ Conference on Human Factors in Computing Systems (Glasgow, Scotland Uk) (CHI '19). Association for Computing Machinery, New York, NY, USA, 1-14. https://doi.org/10.1145/3290605.3300929

[20] Sean G. Gustafson, Bernhard Rabe, and Patrick M. Baudisch. 2013. Understanding Palm-Based Imaginary Interfaces: The Role of Visual and Tactile Cues When Browsing. In Proceedings of the SIGCHI Conference on Human Factors in Computing Systems (Paris, France) (CHI '13). Association for Computing Machinery, New York, NY, USA, 889-898. https://doi.org/10.1145/2470654.2466114

[21] Nur Al-huda Hamdan, Simon Voelker, and Jan Borchers. 2018. Sketch\&Stitch Interactive Embroidery for E-Textiles. In Proceedings of the 2018 CHI Conference on Human Factors in Computing Systems (Montreal QC, Canada) (CHI '18) Association for Computing Machinery, New York, NY, USA, Article 82, 13 pages. https://doi.org/10.1145/3173574.3173656

[22] Nur Al-huda Hamdan, Adrian Wagner, Simon Voelker, Jürgen Steimle, and Jan Borchers. 2019. Springlets: Expressive, Flexible and Silent On-Skin Tactile Interfaces. In Proceedings of the 2019 CHI Conference on Human Factors in Computing Systems (Glasgow, Scotland Uk) (CHI '19). ACM, New York, NY, USA, Article 488, 14 pages. https://doi.org/10.1145/3290605.3300718

[23] Teng Han, Shubhi Bansal, Xiaochen Shi, Yanjun Chen, Baogang Quan, Feng Tian, Hongan Wang, and Sriram Subramanian. 2020. HapBead: On-Skin Microfluidic Haptic Interface Using Tunable Bead. In Proceedings of the 2020 CHI Conference on Human Factors in Computing Systems (Honolulu, HI, USA) (CHI '20). Association for Computing Machinery, New York, NY, USA, 1-10. https://doi.org/10.1145/ 3313831.3376190

[24] Chris Harrison and Haakon Faste. 2014. Implications of Location and Touch for On-Body Projected Interfaces. In Proceedings of the 2014 Conference on Designing Interactive Systems (Vancouver, BC, Canada) (DIS '14). Association for Computing Machinery, New York, NY, USA, 543-552. https://doi.org/10. $1145 / 2598510.2598587$

[25] P. E. Hart, N. J. Nilsson, and B. Raphael. 1968. A Formal Basis for the Heuristic Determination of Minimum Cost Paths. IEEE Transactions on Systems Science and Cybernetics 4, 2 (1968), 100-107.

[26] Steve Hodges, Nicolas Villar, Nicholas Chen, Tushar Chugh, Jie Qi, Diana Nowacka, and Yoshihiro Kawahara. 2014. Circuit Stickers: Peel-and-Stick Construction of Interactive Electronic Prototypes. In Proceedings of the SIGCH Conference on Human Factors in Computing Systems (Toronto, Ontario, Canada) (CHI '14). Association for Computing Machinery, New York, NY, USA, 1743-1746. https://doi.org/10.1145/2556288.2557150

[27] Freehand Ink. 2020. https://inkbox.com/freehand-ink. Accessed: 2020-04-01.

[28] Rotring Isograph. 2020. https://www.rotring.com/de/technische-zeichengerate/ 50-isograph-4006856151008.html. Accessed: 2020-02-15.

[29] Joaquim Jorge and Faramarz Samavati. 2010. Sketch-based interfaces and modeling. Springer Science \& Business Media, Springer-Verlag.

[30] Kalatmak. 2019. Election special Mehndi design / Indian election 2019 henna art Retrieved My 6, 2020 from https://www.youtube.com/watch?v=Qonna8xYGME

[31] Hsin-Liu (Cindy) Kao, Miren Bamforth, David Kim, and Chris Schmandt. 2018 Skinmorph: Texture-tunable On-skin Interface Through Thin, Programmable Gel. In Proceedings of the 2018 ACM International Symposium on Wearable Computers (Singapore, Singapore) (ISWC '18). ACM, New York, NY, USA, 196-203. https://doi.org/10.1145/3267242.3267262

[32] Hsin-Liu (Cindy) Kao, Christian Holz, Asta Roseway, Andres Calvo, and Chris Schmandt. 2016. DuoSkin: Rapidly Prototyping On-skin User Interfaces Using Skin-friendly Materials. In Proceedings of the 2016 ACM International Symposium on Wearable Computers (Heidelberg, Germany) (ISWC '16). ACM, New York, NY,
USA, 16-23. https://doi.org/10.1145/2971763.2971777

[33] Hsin-Liu (Cindy) Kao, Manisha Mohan, Chris Schmandt, Joseph A. Paradiso, and Katia Vega. 2016. ChromoSkin: Towards Interactive Cosmetics Using Thermochromic Pigments. In Proceedings of the $2016 \mathrm{CHI}$ Conference Extended Abstracts on Human Factors in Computing Systems (San Jose, California, USA) (CHI EA '16). ACM, New York, NY, USA, 3703-3706. https://doi.org/10.1145/2851581. 2890270

[34] Yoshihiro Kawahara, Steve Hodges, Benjamin S. Cook, Cheng Zhang, and Gregory D. Abowd. 2013. Instant Inkjet Circuits: Lab-Based Inkjet Printing to Support Rapid Prototyping of UbiComp Devices. In Proceedings of the 2013 ACM International foint Conference on Pervasive and Ubiquitous Computing (Zurich, Switzerland) (UbiComp '13). Association for Computing Machinery, New York, NY, USA, 363-372. https://doi.org/10.1145/2493432.2493486

[35] Arshad Khan, Joan Sol Roo, Tobias Kraus, and Jürgen Steimle. 2019. Soft Inkjet Circuits: Rapid Multi-Material Fabrication of Soft Circuits Using a Commodity Inkjet Printer. In Proceedings of the 32nd Annual ACM Symposium on User Interface Software and Technology (New Orleans, LA, USA) (UIST '19). Association for Computing Machinery, New York, NY, USA, 341-354. https://doi.org/10.1145/3332165.3347892

[36] Soheil Kianzad, Yuxiang Huang, Robert Xiao, and Karon E. MacLean. 2020. Phasking on Paper: Accessing a Continuum of PHysically Assisted SKetchING. In Proceedings of the 2020 CHI Conference on Human Factors in Computing Systems (Honolulu, HI, USA) (CHI '20). Association for Computing Machinery, New York, NY, USA, 1-12. https://doi.org/10.1145/3313831.3376134

[37] Jeonghyun Kim, Anthony Banks, Huanyu Cheng, Zhaoqian Xie, Sheng Xu, Kyung-In Jang, Jung Woo Lee, Zhuangjian Liu, Philipp Gutruf, Xian Huang, Pinghung Wei, Fei Liu, Kan Li, Mitul Dalal, Roozbeh Ghaffari, Xue Feng, Yonggang Huang, Sanjay Gupta, Ungyu Paik, and John A. Rogers. 2015. Epidermal Electronics with Advanced Capabilities in Near-Field Communication. Small 11, 8 (2015), 906-912. https://doi.org/10.1002/smll.201402495

[38] Konstantin Klamka and Raimund Dachselt. 2017. IllumiPaper: Illuminated Interactive Paper. In Proceedings of the 2017 CHI Conference on Human Factors in Computing Systems (Denver, Colorado, USA) (CHI '17). Association for Computing Machinery, New York, NY, USA, 5605-5618. https://doi.org/10.1145/ 3025453.3025525

[39] Konstantin Klamka, Raimund Dachselt, and Jürgen Steimle. 2020. Rapid IronOn User Interfaces: Hands-on Fabrication of Interactive Textile Prototypes. In Proceedings of the 2020 CHI Conference on Human Factors in Computing Systems (Honolulu, HI, USA) (CHI '20). Association for Computing Machinery, New York, NY, USA, 1-14. https://doi.org/10.1145/3313831.3376220

[40] Marion Koelle, Swamy Ananthanarayan, and Susanne Boll. 2020. Social Acceptability in HCI: A Survey of Methods, Measures, and Design Strategies. In Proceedings of the 2020 CHI Conference on Human Factors in Computing Systems (Honolulu, HI, USA) (CHI '20). Association for Computing Machinery, New York, NY, USA, 1-19. https://doi.org/10.1145/3313831.3376162

[41] Lars F. Krutak. 2012. Spiritual Skin: Magical Tattoos and Scarification: Wisdom, Healing, Shamanic Power, Protection. Ed. Reuss, Glattbach.

[42] Emily A. Lai, Eric A. Nesrsta, Jason M. Bodiford, and Ranu R. Jain. 2018. Medical alert tattoos-A pinch of style along with patient safety. Pediatric Anesthesia 28, 12 (2018), 1154-1155. https://doi.org/10.1111/pan.13493

[43] James A. Landay. 1996. SILK: Sketching Interfaces like Krazy. In Conference Companion on Human Factors in Computing Systems (Vancouver, British Columbia, Canada) (CHI '96). Association for Computing Machinery, New York, NY, USA, 398-399. https://doi.org/10.1145/257089.257396

[44] J. A. Landay and B. A. Myers. 2001. Sketching interfaces: toward more human interface design. Computer 34, 3 (2001), 56-64.

[45] Chunyuan Liao, François Guimbretière, and Ken Hinckley. 2005. PapierCraft: A Command System for Interactive Paper. In Proceedings of the 18th Annual ACM Symposium on User Interface Software and Technology (Seattle, WA, USA) (UIST '05). Association for Computing Machinery, New York, NY, USA, 241-244. https://doi.org/10.1145/1095034.1095074

[46] Joanne Lo, Doris Jung Lin Lee, Nathan Wong, David Bui, and Eric Paulos. 2016. Skintillates: Designing and Creating Epidermal Interactions. In Proceedings of the 2016 ACM Conference on Designing Interactive Systems (Brisbane, QLD, Australia) (DIS '16). ACM, New York, NY, USA, 853-864. https://doi.org/10. $1145 / 2901790.2901885$

[47] Joanne Lo, Cesar Torres, Isabel Yang, Jasper O’Leary, Danny Kaufman, Wilmot Li, Mira Dontcheva, and Eric Paulos. 2016. Aesthetic Electronics: Designing, Sketching, and Fabricating Circuits through Digital Exploration. In Proceedings of the 29th Annual Symposium on User Interface Software and Technology (Tokyo, Japan) (UIST '16). Association for Computing Machinery, New York, NY, USA, 665-676. https://doi.org/10.1145/2984511.2984579

[48] Eric Markvicka, Guanyun Wang, Yi-Chin Lee, Gierad Laput, Carmel Majidi, and Lining Yao. 2019. ElectroDermis: Fully Untethered, Stretchable, and HighlyCustomizable Electronic Bandages. In Proceedings of the 2019 CHI Conference on Human Factors in Computing Systems (Glasgow, Scotland Uk) (CHI '19). ACM, New York, NY, USA, Article 632, 10 pages. https://doi.org/10.1145/3290605. 3300862 
[49] David A. Mellis, Sam Jacoby, Leah Buechley, Hannah Perner-Wilson, and Jie Qi 2013. Microcontrollers as Material: Crafting Circuits with Paper, Conductive Ink, Electronic Components, and an "Untoolkit". In Proceedings of the 7th International Conference on Tangible, Embedded and Embodied Interaction (Barcelona, Spain) (TEI '13). Association for Computing Machinery, New York, NY, USA, 83-90. https://doi.org/10.1145/2460625.2460638

[50] Wireless Charging Module. 2020. https://www.seeedstudio.com/WirelessCharging-Module-5V-1A-p-1912.html. Accessed: 2020-09-01.

[51] Stefanie Mueller, Pedro Lopes, and Patrick Baudisch. 2012. Interactive Construc tion: Interactive Fabrication of Functional Mechanical Devices. In Proceedings of the 25th Annual ACM Symposium on User Interface Software and Technology (Cambridge, Massachusetts, USA) (UIST '12). ACM, New York, NY, USA, 599-606. https://doi.org/10.1145/2380116.2380191

[52] Sachith Muthukumarana, Don Samitha Elvitigala, Juan Pablo Forero Cortes, Denys J.C. Matthies, and Suranga Nanayakkara. 2020. Touch Me Gently: Recreating the Perception of Touch Using a Shape-Memory Alloy Matrix. In Proceedings of the 2020 CHI Conference on Human Factors in Computing Systems (Honolulu, HI, USA) (CHI '20). Association for Computing Machinery, New York, NY, USA 1-12. https://doi.org/10.1145/3313831.3376491

[53] Koya Narumi, Steve Hodges, and Yoshihiro Kawahara. 2015. ConductAR: An Augmented Reality Based Tool for Iterative Design of Conductive Ink Circuits In Proceedings of the 2015 ACM International foint Conference on Pervasive and Ubiquitous Computing (Osaka, Japan) (UbiComp '15). Association for Computing Machinery, New York, NY, USA, 791-800. https://doi.org/10.1145/2750858. 2804267

[54] Koya Narumi, Steve Hodges, and Yoshihiro Kawahara. 2015. ConductAR: An Augmented Reality Based Tool for Iterative Design of Conductive Ink Circuits In Proceedings of the 2015 ACM International foint Conference on Pervasive and Ubiquitous Computing (Osaka, Japan) (UbiComp '15). Association for Computing Machinery, New York, NY, USA, 791-800. https://doi.org/10.1145/2750858. 2804267

[55] Aditya Shekhar Nittala, Arshad Khan, Klaus Kruttwig, Tobias Kraus, and Jürgen Steimle. 2020. PhysioSkin: Rapid Fabrication of Skin-Conformal Physiological Interfaces. In Proceedings of the 2020 CHI Conference on Human Factors in Computing Systems (Honolulu, HI, USA) (CHI '20). Association for Computing Machinery, New York, NY, USA, 1-10. https://doi.org/10.1145/3313831.3376366

[56] Aditya Shekhar Nittala, Anusha Withana, Narjes Pourjafarian, and Jürgen Steimle. 2018. Multi-Touch Skin: A Thin and Flexible Multi-Touch Sensor for On-Skin Input. In Proceedings of the 2018 CHI Conference on Human Factors in Computing Systems (Montreal QC, Canada) (CHI '18). ACM, New York, NY, USA, Article 33, 12 pages. https://doi.org/10.1145/3173574.3173607

[57] Hyunjoo Oh, Tung D. Ta, Ryo Suzuki, Mark D. Gross, Yoshihiro Kawahara, and Lining Yao. 2018. PEP (3D Printed Electronic Papercrafts): An Integrated Approach for 3D Sculpting Paper-Based Electronic Devices. In Proceedings of the 2018 CHI Conference on Human Factors in Computing Systems (Montreal QC Canada) (CHI '18). Association for Computing Machinery, New York, NY, USA 1-12. https://doi.org/10.1145/3173574.3174015

[58] Simon Olberding, Sergio Soto Ortega, Klaus Hildebrandt, and Jürgen Steimle. 2015. Foldio: Digital Fabrication of Interactive and Shape-Changing Objects With Foldable Printed Electronics. In Proceedings of the 28th Annual ACM Symposium on User Interface Software and Technology (Charlotte, NC, USA) (UIST '15). Association for Computing Machinery, New York, NY, USA, 223-232. https://doi.org/10.1145/2807442.2807494

[59] Simon Olberding and Jürgen Steimle. 2010. Towards Understanding Erasingbased Interactions: Adding Erasing Capabilities to Anoto Pens. In 1st International Workshop on Paper Computing. PaperComp 2010, Copenhagen, Denmark, 20-23.

[60] M. Packard and E. Kwei. 2012. Henna Sourcebook: Over 1,000 traditional designs and modern interpretations for body decorating. Race Point Publishing, New York. https://books.google.de/books?id=IDeb1a5slLYC

[61] Sunnyscopa Temporary Laser Tattoo Paper. 2020. https://www.amazon.de//en/gp/product/B07FY81ZLG/ref=ppx_yo_dt_b_asin_title_o09_s00?ie=UTF8\& psc $=1$. Accessed: 2020-09-01.

[62] Ncode PDF. 2020. https://www.neosmartpen.com/en/ncode-pdf/. Accessed 2020-09-01.

[63] Huaishu Peng, Jimmy Briggs, Cheng-Yao Wang, Kevin Guo, Joseph Kider, Stefanie Mueller, Patrick Baudisch, and François Guimbretière. 2018. RoMA: Interactive Fabrication with Augmented Reality and a Robotic 3D Printer. In Proceedings of the 2018 CHI Conference on Human Factors in Computing Systems (Montreal QC, Canada) (CHI '18). Association for Computing Machinery, New York, NY, USA, Article 579, 12 pages. https://doi.org/10.1145/3173574.3174153

[64] Huaishu Peng, Amit Zoran, and François V. Guimbretière. 2015. D-Coil: A Handson Approach to Digital 3D Models Design. In Proceedings of the 33rd Annual ACM Conference on Human Factors in Computing Systems (Seoul, Republic of Korea) (CHI '15). Association for Computing Machinery, New York, NY, USA 1807-1815. https://doi.org/10.1145/2702123.2702381

[65] Hao-Lun Peng and Yoshihiro Watanabe. 2020. High-Speed Human Arm Projection Mapping with Skin Deformation. In SIGGRAPH Asia 2020 Emerging
Technologies (Virtual Event, Republic of Korea) (SA '20). Association for Computing Machinery, New York, NY, USA, Article 13, 2 pages. https://doi.org/10. $1145 / 3415255.3422887$

[66] E. R. Post, M. Orth, P. R. Russo, and N. Gershenfeld. 2000. E-broidery: Design and fabrication of textile-based computing. IBM Systems fournal 39, 3.4 (2000), $840-860$.

[67] Narjes Pourjafarian, Anusha Withana, Joseph A. Paradiso, and Jürgen Steimle. 2019. Multi-Touch Kit: A Do-It-Yourself Technique for Capacitive Multi-Touch Sensing Using a Commodity Microcontroller. In Proceedings of the 32nd Annual ACM Symposium on User Interface Software and Technology (New Orleans, LA, USA) (UIST '19). Association for Computing Machinery, New York, NY, USA, 1071-1083. https://doi.org/10.1145/3332165.3347895

[68] Prinker. 2020. https://prinker-europe.com/en/. Accessed: 2020-02-15

[69] The Tattoo Archive Project. 2020. https://artsandculture.google.com/exhibit/ history-ink/wQx72HUG. Accessed: 2020-04-01.

[70] Bridget Punsalang. 2011. How to Henna a Pregnant Belly, Workshop at the 4th annual Henna Gathering. https://www.youtube.com/watch?v=lBAXrNRs9Ek Accessed: 2020-09-01.

[71] Jie Qi and Leah Buechley. 2010. Electronic Popables: Exploring Paper-Based Computing through an Interactive Pop-up Book. In Proceedings of the Fourth International Conference on Tangible, Embedded, and Embodied Interaction (Cambridge, Massachusetts, USA) (TEI '10). Association for Computing Machinery, New York, NY, USA, 121-128. https://doi.org/10.1145/1709886.1709909

[72] Jie Qi and Leah Buechley. 2014. Sketching in Circuits: Designing and Building Electronics on Paper. In Proceedings of the SIGCHI Conference on Human Factors in Computing Systems (Toronto, Ontario, Canada) (CHI '14). ACM, New York, NY, USA, 1713-1722. https://doi.org/10.1145/2556288.2557391

[73] Raf Ramakers, Kashyap Todi, and Kris Luyten. 2015. PaperPulse: An Integrated Approach for Embedding Electronics in Paper Designs. In Proceedings of the 33rd Annual ACM Conference on Human Factors in Computing Systems (Seoul, Republic of Korea) (CHI '15). ACM, New York, NY, USA, 2457-2466. https: //doi.org/10.1145/2702123.2702487

[74] Maya Ranganathan and Bernadette Lobo. 2008. Localizing the global: Analysis of nationalist ideologies in MNC advertisements in Indian TV. Nationalism and Ethnic Politics 14, 1 (2008), 117-142.

[75] Michael Rees. 2016. From Outsider to Established-Explaining the Current Popularity and Acceptability of Tattooing. Historical Social Research/Historische Sozialforschung 41, 3 (2016), 157-174.

[76] Beate Reiser, Tobias Kraus, Lola Gonzalez-Garcia, Johannes H.M. Maurer, and Ioannis Kanelidis. 2017. Conductive nanocomposites. Retrieved 15.04.2020 from https:/www.leibniz-inm.de/wp-content/uploads/Handout-Sintering-freeink.pdf Patent No. WO2017045989A1, Filed September 8th., 2016, Issued March 23th., 2017.

[77] Alec Rivers, Ilan E. Moyer, and Frédo Durand. 2012. Position-Correcting Tools for 2D Digital Fabrication. ACM Trans. Graph. 31, 4, Article 88 (July 2012), 7 pages. https://doi.org/10.1145/2185520.2185584

[78] Thijs Roumen, Bastian Kruck, Tobias Dürschmid, Tobias Nack, and Patrick Baudisch. 2016. Mobile Fabrication. In Proceedings of the 29th Annual Symposium on User Interface Software and Technology (Tokyo, Japan) (UIST '16). Association for Computing Machinery, New York, NY, USA, 3-14. https://doi.org/10.1145/ 2984511.2984586

[79] Jyoti Sachdeva. 2018. Valentine's Day Special Arabic Henna Mehndi Designs with Cookie Cutter. https://www.youtube.com/watch?v=YFNPrz-soLU Accessed: 2020-09-01.

[80] Jogendra Saksena. 1977. Henna for happiness; India's "mehndi" art of symbols for all seasons. The UNESCO Courier: $a$ window open on the world XXX, 2 (1977), 18-22. https://unesdoc.unesco.org/ark:/48223/pf0000046525

[81] Valkyrie Savage, Xiaohan Zhang, and Björn Hartmann. 2012. Midas: Fabricating Custom Capacitive Touch Sensors to Prototype Interactive Objects. In Proceedings of the 25th Annual ACM Symposium on User Interface Software and Technology (Cambridge, Massachusetts, USA) (UIST'12). Association for Computing Machinery, New York, NY, USA, 579-588. https://doi.org/10.1145/2380116.2380189

[82] Eldon Schoop, Michelle Nguyen, Daniel Lim, Valkyrie Savage, Sean Follmer, and Björn Hartmann. 2016. Drill Sergeant: Supporting Physical Construction Projects through an Ecosystem of Augmented Tools. In Proceedings of the 2016 CHI Conference Extended Abstracts on Human Factors in Computing Systems (San Jose, California, USA) (CHIEA '16). Association for Computing Machinery, New York, NY, USA, 1607-1614. https://doi.org/10.1145/2851581.2892429

[83] Circuit Scribe. 2020. https://circuitscribe.com/. Accessed: 2020-08-15.

[84] Ronald WB Scutt and Christopher Gotch. 1974. Art, sex and symbol: The mystery of tattooing. AS Barnes, South Brunswick.

[85] Roy Shilkrot, Pattie Maes, Joseph A. Paradiso, and Amit Zoran. 2015. Augmented Airbrush for Computer Aided Painting (CAP). ACM Trans. Graph. 34, 2, Article 19 (March 2015), 11 pages. https://doi.org/10.1145/2699649

[86] Neo Smartpen. 2020. https://www.neosmartpen.com/. Accessed: 2020-02-15.

[87] Hyunyoung Song, Tovi Grossman, George Fitzmaurice, François Guimbretiere, Azam Khan, Ramtin Attar, and Gordon Kurtenbach. 2009. PenLight: Combining a Mobile Projector and a Digital Pen for Dynamic Visual Overlay. In Proceedings 
of the SIGCHI Conference on Human Factors in Computing Systems (Boston, MA USA) (CHI '09). Association for Computing Machinery, New York, NY, USA 143-152. https://doi.org/10.1145/1518701.1518726

[88] J. Steimle, J. Bergstrom-Lehtovirta, M. Weigel, A. S. Nittala, S. Boring, A. Olwal, and K. Hornbæk. 2017. On-Skin Interaction Using Body Landmarks. Computer 50, 10 (2017), 19-27.

[89] Paul Strohmeier, Juan Pablo Carrascal, and Kasper Hornbæk. 2016. What Can Doodles on the Arm Teach Us About On-Body Interaction?. In Proceedings of the 2016 CHI Conference Extended Abstracts on Human Factors in Computing Systems (San Jose, California, USA) (CHI EA '16). ACM, New York, NY, USA 2726-2735. https://doi.org/10.1145/2851581.2892544

[90] Haruki Takahashi and Jeeeun Kim. 2019. 3D Pen + 3D Printer: Exploring the Role of Humans and Fabrication Machines in Creative Making. In Proceedings of the 2019 CHI Conference on Human Factors in Computing Systems (Glasgow, Scotland Uk) (CHI '19). ACM, New York, NY, USA, Article 295, 12 pages. https: //doi.org/10.1145/3290605.3300525

[91] Katia Vega, Abel Arrieta, Felipe Esteves, and Hugo Fuks. 2014. FX e-Makeup for Muscle Based Interaction. In Design, User Experience, and Usability. User Experience Design for Everyday Life Applications and Services, Aaron Marcus (Ed.). Springer International Publishing, Cham, 643-652.

[92] Katia Vega and Hugo Fuks. 2014. Beauty Technology: Body Surface Computing. Computer 47, 4 (April 2014), 71-75. https://doi.org/10.1109/MC.2014.81

[93] Katia Vega, Nan Jiang, Xin Liu, Viirj Kan, Nick Barry, Pattie Maes, Ali K. Yetisan, and Joe Paradiso. 2018. The Dermal Abyss: Color-Changing Tattoos for Medical Diagnostics. GetMobile: Mobile Comp. and Comm. 22, 2 (Sept. 2018), 38-42. https://doi.org/10.1145/3276145.3276158

[94] Artie Venti. 2019. How Do Tattoo Artists Transfer Designs from Drawing to Skin? https://readinsideout.com/education/how-do-tattoo-artists-transferdesigns-from-drawing-to-skin/

[95] Yanan Wang, Shijian Luo, Yujia Lu, Hebo Gong, Yexing Zhou, Shuai Liu, and Preben Hansen. 2017. AnimSkin: Fabricating Epidermis with Interactive, Functional and Aesthetic Color Animation. In Proceedings of the 2017 Conference on Designing Interactive Systems (Edinburgh, United Kingdom) (DIS '17). ACM, New York, NY, USA, 397-401. https://doi.org/10.1145/3064663.3064687

[96] Ian Watts. 2009. Red ochre, body painting, and language: interpreting the Blombos ochre. The cradle of language 2 (2009), 93-129.

[97] Martin Weigel, Tong Lu, Gilles Bailly, Antti Oulasvirta, Carmel Majidi, and Jürgen Steimle. 2015. iSkin: Flexible, Stretchable and Visually Customizable OnBody Touch Sensors for Mobile Computing. In Proceedings of the 33rd Annual ACM Conference on Human Factors in Computing Systems (Seoul, Republic of Korea) (CHI '15). ACM, New York, NY, USA, 2991-3000. https://doi.org/10.1145/ 2702123.2702391

[98] Martin Weigel, Vikram Mehta, and Jürgen Steimle. 2014. More than Touch: Understanding How People Use Skin as an Input Surface for Mobile Computing In Proceedings of the SIGCHI Conference on Human Factors in Computing Systems (Toronto, Ontario, Canada) (CHI '14). Association for Computing Machinery, New York, NY, USA, 179-188. https://doi.org/10.1145/2556288.2557239

[99] Martin Weigel, Aditya Shekhar Nittala, Alex Olwal, and Jürgen Steimle. 2017. SkinMarks: Enabling Interactions on Body Landmarks Using Conformal Skin Electronics. In Proceedings of the 2017 CHI Conference on Human Factors in Computing Systems (Denver, Colorado, USA) (CHI '17). Association for Computing Machinery, New York, NY, USA, 3095-3105. https://doi.org/10.1145/3025453. 3025704

[100] Cliff White. 2016. Classic Tattoo Stencils 2: More Designs in Acetate. Number no. 2 in 1. Schiffer Publishing Limited, Pennsylvania, USA. https://books.google. de/books?id=pP6-DAEACAAJ
[101] Karl D.D. Willis, Cheng Xu, Kuan-Ju Wu, Golan Levin, and Mark D. Gross. 2011. Interactive Fabrication: New Interfaces for Digital Fabrication. In Proceedings of the Fifth International Conference on Tangible, Embedded, and Embodied Interaction (Funchal, Portugal) (TEI '11). ACM, New York, NY, USA, 69-72. https://doi.org/10.1145/1935701.1935716

[102] Anusha Withana, Daniel Groeger, and Jürgen Steimle. 2018. Tacttoo: A Thin and Feel-Through Tattoo for On-Skin Tactile Output. In Proceedings of the 31st Annual ACM Symposium on User Interface Software and Technology (Berlin, Germany) (UIST '18). ACM, New York, NY, USA, 365-378. https://doi.org/10. $1145 / 3242587.3242645$

[103] Jun Xie, Aaron Hertzmann, Wilmot Li, and Holger Winnemöller. 2014. PortraitSketch: Face Sketching Assistance for Novices. In Proceedings of the 27th Annual ACM Symposium on User Interface Software and Technology (Honolulu, Hawaii, USA) (UIST '14). Association for Computing Machinery, New York, NY, USA, 407-417. https://doi.org/10.1145/2642918.2647399

[104] Junichi Yamaoka and Yasuaki Kakehi. 2013. DePENd: Augmented Handwriting System Using Ferromagnetism of a Ballpoint Pen. In Proceedings of the 26th Annual ACM Symposium on User Interface Software and Technology (St. Andrews, Scotland, United Kingdom) (UIST '13). Association for Computing Machinery, New York, NY, USA, 203-210. https://doi.org/10.1145/2501988.2502017

[105] Sang Ho Yoon, Siyuan Ma, Woo Suk Lee, Shantanu Thakurdesai, Di Sun, Flávio P. Ribeiro, and James D. Holbery. 2019. HapSense: A Soft Haptic I/O Device with Uninterrupted Dual Functionalities of Force Sensing and Vibrotactile Actuation. In Proceedings of the 32nd Annual ACM Symposium on User Interface Software and Technology (New Orleans, LA, USA) (UIST '19). Association for Computing Machinery, New York, NY, USA, 949-961. https://doi.org/10.1145/3332165. 3347888

[106] Chuang-Wen You, Min-Wei Hung, Ximeng Zhang, Po-Chun Huang, and HsinLiu (Cindy) Kao. 2020. Online Survey Study on Social Perceptions towards ColorChanging on-Skin Displays. In Proceedings of the 2020 International Symposium on Wearable Computers (Virtual Event, Mexico) (ISWC '20). Association for Computing Machinery, New York, NY, USA, 90-95. https://doi.org/10.1145/ 3410531.3414301

[107] Chuang-Wen You, Ya-Fang Lin, Elle Luo, Hung-Yeh Lin, and Hsin-Liu (Cindy) Kao. 2019. Understanding Social Perceptions towards Interacting with OnSkin Interfaces in Public. In Proceedings of the 23rd International Symposium on Wearable Computers (London, United Kingdom) (ISWC '19). Association for Computing Machinery, New York, NY, USA, 244-253. https://doi.org/10.1145/ 3341163.3347751

[108] Ya-Ting Yue, Xiaolong Zhang, Yongliang Yang, Gang Ren, Yi-King Choi, and Wenping Wang. 2017. WireDraw: 3D Wire Sculpturing Guided with Mixed Reality. In Proceedings of the 2017 CHI Conference on Human Factors in Computing Systems (Denver, Colorado, USA) (CHI '17). Association for Computing Machinery, New York, NY, USA, 3693-3704. https://doi.org/10.1145/3025453.3025792

[109] Zhijie Zhu, Shuang-Zhuang Guo, Tessa Hirdler, Cindy Eide, Xiaoxiao Fan, Jakub Tolar, and Michael C. McAlpine. 2018. 3D Printed Functional and Biological Materials on Moving Freeform Surfaces. Advanced Materials 30, 23 (2018), 1707495. https://doi.org/10.1002/adma.201707495

[110] Amit Zoran and Joseph A. Paradiso. 2013. FreeD: A Freehand Digital Sculpting Tool. In Proceedings of the SIGCHI Conference on Human Factors in Computing Systems (Paris, France) (CHI '13). ACM, New York, NY, USA, 2613-2616. https: //doi.org/10.1145/2470654.2481361

[111] Amit Zoran, Roy Shilkrot, and Joseph Paradiso. 2013. Human-Computer Interaction for Hybrid Carving. In Proceedings of the 26th Annual ACM Symposium on User Interface Software and Technology (St. Andrews, Scotland, United Kingdom) (UIST '13). Association for Computing Machinery, New York, NY, USA, 433-440. https://doi.org/10.1145/2501988.2502023 